\title{
Containment Control of Underactuated Ships with Environment Disturbances and Parameter Uncertainties
}

\author{
Qidan Zhu, ${ }^{1}$ Junda Ma, ${ }^{1}$ Zhilin Liu, ${ }^{1}$ and Ke Liu ${ }^{2}$ \\ ${ }^{1}$ College of Automation, Harbin Engineering University, Harbin 150001, China \\ ${ }^{2}$ College of Engineering, Temple University, Philadelphia, PA 19122, USA \\ Correspondence should be addressed to Junda Ma; majunda2016@163.com
}

Received 27 December 2016; Revised 19 February 2017; Accepted 6 March 2017; Published 2 April 2017

Academic Editor: Tomasz Kapitaniak

Copyright (C) 2017 Qidan Zhu et al. This is an open access article distributed under the Creative Commons Attribution License, which permits unrestricted use, distribution, and reproduction in any medium, provided the original work is properly cited.

An implementable robust containment control algorithm is proposed for a group of underactuated ships in the presence of hydrodynamic parameter uncertainties and external disturbances. The control objective is to drive all the followers into the convex hull spanned by the virtual leaders, whose state information is available only to a subset of the followers. For this purpose, the ship model is primarily transformed to a strict-feedback form. In the kinematic design, a virtual containment controller, requiring the state information from its neighbors, is presented based on the results obtained from graph theory. In the dynamic design, a robust containment controller is developed through utilizing upper-to-up sliding mode control. In addition, in order to simplify the implementations of the control law, the command filtered backstepping (CFBP) method is introduced to prevent the analytic differentiations of the virtual law from each design step of the backstepping (BP) method. Subsequently, it is well proven that all the tracking errors could converge to and remain small neighborhoods of the equilibrium point. Finally, several simulation experiments are conducted to demonstrate the performance of the proposed control algorithm.

\section{Introduction}

Over the past few years, a huge and rapidly growing body of research has focused on the cooperative control of the multiple vehicle systems within a motion-control community because of their broad applications, for example, wheeled mobile robot, manipulator, spacecraft, and ship [1-4]. Although an individual vehicle can be used to complete a specific task, some benefits including greater robustness, lower cost, and better performance can be achieved through a group of vehicles working cooperatively. In the marine domain, many applications using cooperative control include various tasks such as environmental monitoring as well as rescue and reconnaissance operations [4-7]. To perform these tasks, an increasing number of studies have focused on the cooperative control of multiple ships.

These studies have included behavior-based method [8], virtual structure method [9-14], and leader-follower (L-F) method $[4,5,15-19]$. On the basis of null space methods, a formation control strategy has been developed to achieve multiple tasks in [8]. In [15], an output synchronization control strategy has been designed for the L-F control of ships. The authors in $[9,10]$ introduce passivity theory as a practical design tool for solving the formation problem, which includes group coordination and path following. A robust cooperative control method has also been applied to form a desired geometric pattern with the aid of a neural network $(\mathrm{NN})$, a backstepping (BP) method, and graph theory [11]. In [8-11, 15], the vehicles are assumed to be fully actuated.

In actuality, most ships are underactuated, meaning that they are not actuated in the sway axis [20]. Since the dynamics of each underactuated ship do not satisfy the necessary conditions of Brockett for stabilization [21], the cooperative control law for each ship cannot be smooth functions with theirs variables [16]. In [12], a decentralized formation control method has been presented to address such an issue by using the nonlinear cascaded system theory and line-of-sight guidance to ensure a straight-line path following for the formations of ships. On the basis of Lyapunov theory, the BP method, and the graph theory, a path-following coordination controller 
(PFCC) is proposed in [13] and its control strategy also considers time delayed communication among ships. The work of [14] is extended further in [13]. In [14], a dynamic surface control (DSC) technique is proposed to estimate the virtual control at each step of the BP method. An additional virtual control is adopted to solve the difficult problems when designing the formation controller for ships in [22]. The LF method for ship has been also reported in [4, 5, 16-19]. In $[16,17]$, continuous time-varying cooperative control laws are designed to perform a geometric pattern by using suitable transformations. It is noted that, in $[16,17]$, the yaw velocity is assumed to be nonzero, which is referred to as the "persistent excitation" (PE) condition. Under this condition, a straight line cannot be tracked for underactuated ships. The authors in [18] address the design of the nonlinear model predictive formation controller, where the relative distances and orientations between the follower and the leader can be stabilized. To cope with the uncertainties of the model, two different robust L-F formation control laws have been designed that combine NN with the DSC technique in $[4,19]$. More recently, a robust formation control algorithm is proposed to force ships to maintain the desired orientations and positions relative to one leading vessel, considering the limited magnitude of the control signal in [5]. In $[4,5,19]$, the radial basis function $\mathrm{NN}$ and adaptive control method can approximate the nonlinear uncertainty systems, which increases the complexity of the online computing. In addition, in all the works on L-F formation control, a common trait is that only one leader exists in the group.

In practical applications, for a group of vehicles multiple leaders might exist. In this case, the control objective is to drive the followers of the group into the convex hull spanned by the leaders, which is called the containment control problem [23]. Thus, containment control can be regarded as a special L-F formation control, and the study of containment control is motivated by its possible applications. For example, a group of ships are guided by another group, and some ships are regarded as leaders, which are all equipped with sensors to detect the hazardous obstacles in the marine environment [24]. As such, the leaders can form a safe area in which the followers can converge [25]. Recent works on the containment control of multiple vehicle systems focus on single integrator systems [25], double integrator systems [26], strictfeedback form systems [23], general linear dynamics [27], and Lagrange dynamics [28]. However, these proposed methods cannot be applied directly to an underactuated mechanical system, and the uncertainties from the model might affect the control performance. Accordingly, how to achieve the containment control objective in the presence of uncertain dynamics and external disturbances needs to be investigated further.

For nonlinear systems, various methods have been adopted, and, among these, the BP method can be regarded as a major design tool. However, this method suffers from the "explosion of complexity" problem, which is caused by the repeated derivatives of the virtual control signals [29]. To overcome this drawback, the command filtered backstepping
(CFBP) method, which introduces, at each design step of the BP method, a command filter to prevent the derivative of nonlinear functions, has also been applied to the strict-feedback form system in [30, 31]. The CFBP method is regarded as an improved version of standard BP method and is widely used in different fields [32-34]. However, these works cannot prove the boundedness of the tracking errors, which implies that the stability of the closed-loop system cannot be analyzed quantifiably. In addition, most current CFBP adopted second-order command filter (SOCF), and the firstorder command filter (FOCF) with simpler structure is rarely mentioned.

In conclusion, the containment control of underactuated ships involved the following main difficulties: (1) the underactuated surface ship lacks control input at side, so it belongs to a class of underactuated system; namely, the general nonlinear control theory could not be directly applied; (2) in complex sea cases, ship is often influenced by the external disturbances and parameter uncertainties (EDPU), which makes it complicated to accurately control the motion of ship; (3) the current research results on containment control are applied only in full-actuated system such as single integrator systems, double integrator systems, and Lagrange systems. However, it is not mentioned how to achieve containment control by combining the knowledge of graph theory and the underactuated system, which is one of the research difficulties. In this paper, a robust containment control strategy, inspired by the previous works, which is performed by using the standard BP method, the FOCF technique, the sliding mode control (SMC) method, the Lyapunov stability theory, and the results from graph theory, is developed for a group of ships in the presence of the EDPU. Compared with the existing results, the main features of this paper can be summarized as follows: (1) for the first time, this paper considers the containment problem of underactuated ships; (2) by incorporating the FOCF technique, the $\mathrm{BP}$ commands are simplified; (3) different from the previous conclusions about SOCF [30, 32-34], the stability of all the closed-loop systems is first analyzed quantifiably; (4) by introducing a polar coordinate, the model is transformed to a strict- feedback form system, and the PE condition from [16,17] is avoided; (5) compared with the results in $[4,5,19]$, the development, using upper-toup SMC to design a robust containment controller, is more practical because the online computation of the uncertainties is avoided.

\section{Control Problem Formulation}

2.1. Notations. To prepare for the subsequent control design, some notations are standard as below: $\mathbf{R}^{n \times m}$ denotes a set of Euclidean $n \times m$ matrices, and $\mathbf{R}^{n}$ denotes $n$-dimensional Euclidean space; $\operatorname{diag}\left(a_{1}, a_{2}, \ldots, a_{n}\right)$ denotes a diagonal matrix with entries $a_{i}(i=1,2, \ldots, n)$; the maximum and the minimum eigenvalues of a square matrix are described as $\lambda_{\max }(\cdot)$ and $\lambda_{\text {min }}(\cdot)$, respectively; let $|\cdot|$ be the absolute value of a scalar; let $\|\cdot\|$ be the Euclidean norm of a vector; let $\mathbf{A} \otimes \mathbf{B}$ be the Kronecker product of matrices $\mathbf{A} \in \mathbf{R}^{n \times m}$ and $\mathbf{B} \in \mathbf{R}^{p \times q}$; $C:=D$ denotes that $D$ is defined as $C$. 
2.2. Concepts in Graph Theory. Suppose that a group of under actuated ships interact with each other through a communication network to perform a containment control task. It is natural to model the communication topology among these ships by using graph theory. Without loss of generality, we assume that the individual ship is a node, and the interaction for ships can be described by a directed graph $G=\{V, \varepsilon\}$, where $V=\left\{a_{1}, a_{2}, \ldots, a_{n}\right\}$ denotes a set of nodes and $\varepsilon=$ $\left\{\left(n_{i}, n_{j}\right) \in V \times V\right\}$ denotes a set of edges with element $\left(n_{i}, n_{j}\right)$, which implies that node $n_{j}$ can receive information from node $n_{i}$. Here, we say that node $n_{i}$ is a neighbor of node $n_{j}$, and the notation $N_{i}$ is described as the set of all neighbors of node. Let the adjacency matrix $\mathbf{A}=\left\{a_{i j}\right\} \in \mathbf{R}^{N \times N}$ be defined such as $a_{i j}=1$ if $\left(n_{i}, n_{j}\right) \in \varepsilon$ and $a_{i j}=0$ otherwise; Note that we assume $a_{i i}=0$ for all the nodes. The Laplacian matrix $\mathbf{L}=\left\{l_{i j}\right\}$ of the directed graph $G$ is defined such that

$$
l_{i j}=\sum_{j=1}^{N} a_{i j} \quad \text { if } j=i ; l_{i j}=-a_{i j} \text {, otherwise. }
$$

A directed path from $n_{i}$ to $n_{j}$ in the graph $G$ is a set of edges: $\left(n_{i}, n_{i+1}\right),\left(n_{i+1}, n_{i+2}\right), \ldots,\left(n_{j-2}, n_{j-1}\right)$, and $\left(n_{j-1}, n_{j}\right)$, where all the nodes in this path are different [35].

Definition 1. For a group of ships, ship $i$ is said to be a leader if $a_{i j}=0$, and ship $i$ is said to be a follower if $a_{i j}=1, j=$ $1,2, \ldots, N$.

Definition 2. The real set $C \in \mathbf{R}^{n}$ is said to be convex if, $\forall x, y \in C$, there exists a point that satisfies $(1-z) x+z y \in C$ for any $z \in[0,1]$. The convex hull for a set of points $X=$ $\left\{x_{1}, x_{2}, \ldots, x_{N}\right\}$ in $C$ is the minimal convex set including all the points in $X$ and let $\operatorname{Co}(X)$ be the convex hull of $X$. In particular, $\operatorname{Co}(X)$ is defined as follows [36]:

$$
\operatorname{Co}(X)=\left\{\sum_{i=1}^{n} \lambda_{i} x_{i} \mid x_{i} \in X, \lambda_{i}>0, \sum_{i=1}^{n} \lambda_{i}=1\right\} .
$$

2.3. Underactuated Ship Modeling. Suppose that there are $M$ identical followers, labeled as ship 1 to $M$. According to [37], for the containment control task we can neglect the motions in heave, pitch, and roll; hence, for a ship, the 3-DOF mathematical model can be formulated as (see Figure 1)

$$
\begin{aligned}
& \dot{x}_{i}=u_{i} \cos \psi_{i}-v_{i} \sin \psi_{i}, \\
& \dot{y}_{i}=u_{i} \cos \psi_{i}+v_{i} \sin \psi_{i}, \\
& \dot{\psi}_{i}=r_{i}, \\
& \dot{u}_{i}=f_{u i}\left(u_{i}, v_{i}, r_{i}\right)+m_{11 i}^{-1}\left(F_{u i}+\tau_{w u i}(t)\right), \\
& \dot{v}_{i}=f_{v i}\left(u_{i}, v_{i}, r_{i}\right)+m_{22 i}^{-1} \tau_{w v i}(t), \\
& \dot{r}_{i}=f_{r i}\left(u_{i}, v_{i}, r_{i}\right)+m_{33 i}^{-1}\left(F_{r i}+\tau_{w r i}(t)\right),
\end{aligned}
$$

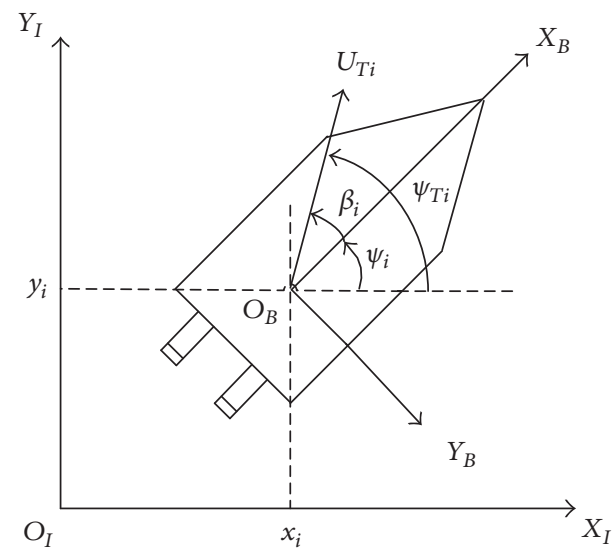

FIgURE 1: Planar model of an underactuated ship.

where

$$
\begin{aligned}
f_{u i}= & m_{22 i} m_{11 i}^{-1} v_{i} r_{i}-d_{u i} m_{11 i}^{-1} u_{i}-d_{u 2 i} m_{11 i}^{-1}\left|u_{i}\right| u_{i} \\
f_{v i}= & -m_{11 i} m_{22 i}^{-1} u_{i} r_{i}-d_{v i} m_{22 i}^{-1} v_{i}-d_{v 2 i} m_{22 i}^{-1}\left|v_{i}\right| v_{i} \\
& -d_{v r i} m_{22 i}^{-1} r_{i} \\
f_{r i}= & \left(m_{11 i}-m_{22 i}\right) m_{33 i}^{-1} u_{i} v_{i}-d_{r i} m_{33 i}^{-1} r_{i} \\
& -d_{r 2 i} m_{33 i}^{-1}\left|r_{i}\right| r_{i}-d_{v r i} m_{22 i}^{-1} r_{i},
\end{aligned}
$$

where $\left(x_{i}, y_{i}\right)$ and $\psi_{i}$ denote the position and heading angle of the ship $i$ in the inertial reference frame $\{I\} ; u_{i}, v_{i}$, and $r_{i}$ denote surge, sway, and yaw velocities expressed in the vessel-fixed reference frame $\{B\}$, respectively; $\tau_{w u i}, \tau_{w v i}$, and $\tau_{w r i}$ describe ocean environment disturbances; $F_{u i}$ and $F_{r i}$ are used to describe actual control inputs; $d_{u i}, d_{u 2 i}, d_{v i}, d_{v 2 i}, d_{v r i}$, $d_{r i}, d_{r 2 i}$, and $d_{r v i}$ denote hydrodynamic damping parameters; $m_{11 i}, m_{22 i}$, and $m_{33 i}$ denote ship inertia including added mass.

Because no control signal is introduced in the sway direction, this model is an underactuated system. Inspired by the work of [38], we define the following polar coordinates to solve this problem:

$$
\begin{aligned}
& U_{T i}=\sqrt{u_{i}^{2}+v_{i}^{2}}, \\
& \psi_{T i}=\psi_{i}+\beta_{i},
\end{aligned}
$$

where $\beta_{i}=\arctan \left(v_{i}, u_{i}\right)$ is often recognized as side-angle and $U_{T i}$ and $\psi_{T i}$ are recognized as the speed and the course angle of ship $i$. Then, substituting (5) into (3) yields

$$
\begin{aligned}
\dot{x}_{i}= & U_{T i} \cos \psi_{T i}, \\
\dot{y}_{i}= & U_{T i} \sin \psi_{T i}, \\
\dot{\psi}_{T i}= & r_{i}+\dot{\beta}_{i}, \\
\dot{V}_{T i}= & \cos \beta_{i} f_{u i}+\sin \beta_{i} f_{v i}+\cos \beta_{i} m_{11 i}^{-1} \tau_{w u i}(t) \\
& +\sin \beta_{i} m_{22 i}^{-1} \tau_{w v i}(t)+\cos \beta_{i} m_{11 i}^{-1} F_{u i}, \\
\dot{r}_{i}= & f_{r i}+m_{33 i}^{-1} \tau_{w r i}(t)+m_{33 i}^{-1} F_{r i}(t) .
\end{aligned}
$$


Then, suppose that the group contains $N-M$ leaders in the group, labeled as leaders $M+1$ to $N$. To describe the communication among the followers and leaders in the group, let $\mathbf{L}$ denote the Laplacian matrix. As each leader has no neighbors, $\mathbf{L}$ can be described by the following:

$$
\mathbf{L}=\left[\begin{array}{cc}
\mathbf{L}_{1} & \mathbf{L}_{2} \\
\mathbf{0}_{(N-M) \times M} & \mathbf{0}_{(N-M) \times(N-M)}
\end{array}\right],
$$

where $\mathbf{L}_{1} \in \mathbf{R}^{M \times M}$ and $\mathbf{L}_{2} \in \mathbf{R}^{M \times(N-M)}$.

Assumption 3. The environment disturbances and $\tau_{w u i}, \tau_{w v i}$, and $\tau_{w r i}$ satisfy

$$
\begin{aligned}
& \left|\tau_{w u i}\right| \leq \bar{\tau}_{w u i}, \\
& \left|\tau_{w v i}\right| \leq \bar{\tau}_{w v i}, \\
& \left|\tau_{w r i}\right| \leq \bar{\tau}_{w r i},
\end{aligned}
$$

where $\bar{\tau}_{w u i}, \bar{\tau}_{w v i}, \bar{\tau}_{w r i}>0[38]$.

Assumption 4. For every follower of the group, there exists at least one leader that has a directed path to it [36].

Assumption 5. The trajectories of the virtual leaders $\phi_{i}=$ $\left[x_{d i}, y_{d i}\right]^{T}(i=M+1, \ldots, N)$ satisfy

$$
\begin{aligned}
0 & <b_{1 i} \leq\left\|\dot{\phi}_{i}\right\| \leq b_{2 i}, \\
\left\|\ddot{\phi}_{i}\right\| & \leq b_{3 i},
\end{aligned}
$$

where $b_{1 i}, b_{2 i}$, and $b_{3 i}$ are all positive constants.

Remark 6. (1) Assumption 3 means that the disturbances induced by winds, waves, and currents have finite energy; (2) Assumption 4 indicates that the proposed control law relies just on a directed communication topology, which reduces the complexity of the communication among ships; (3) the boundedness of the first derivatives and second derivatives of the leaders will be introduced to analyze the stability of the closed-loop system.

Remark 7. Because the surge control $F_{u i}$ in (7) is available only for the nonzero value of $\cos \beta_{i}$, the condition $U_{T i}>0$ can guarantee that the side-angle satisfies $\beta_{i} \neq \pm 0.5 \pi$; that is, $\cos \beta_{i} \neq 0$. In the next part, we will give a certain initial condition for ship $i$ such that $U_{T i}$ is positive at all times.

Remark 8. Using the polar coordinate transformation (5), the underactuated models (3) are transformed to a strictfeedback form of (6) and (7). Then, one can apply the standard BP method to design the control input signals $F_{u i}$ and $F_{r i}$, which achieves the containment control objective.

2.4. Command Filter. To overcome the problem of "explosion of complexity" which occurs in a strict-feedback form [39], a FOCF is introduced to eliminate the analytic differentiation of the virtual control signal in the standard BP design procedure [30]. The standard state space form of FOCF is presented as

$$
\dot{z}=-\omega_{n}(z-q),
$$

where the FOCF parameter $\omega_{n}$ is a constant and satisfies $\omega_{n}>0 ; z$ and $\dot{z}$ are estimates of the input $q$ and its derivative, respectively; the initial conditions of states $z$ and $z-q$ are $\lambda_{3}$ and $\lambda_{4}$; the following lemma proves the input-to-state stability property of FOCF which will be used in Section 3 to analyze the stability of the closed-loop system.

Lemma 9. Consider the FOCF described by (11). Assume that the positive constants $\lambda_{1}$ and $\lambda_{2}$ exist, such that $|q(t)| \leq \lambda_{1}$, $|\dot{q}(t)| \leq \lambda_{2}$ for all $t \geq 0$. Then, the following properties of the FOCF can be given:

(1) $z_{1}$ is bounded and satisfies

$$
|z| \leq \lambda_{1}+\left|\lambda_{3}-\lambda_{4}\right|+\frac{\lambda_{1}}{\omega_{n}} .
$$

(2) $\dot{z}_{1}$ is bounded and satisfies

$$
|\dot{z}| \leq \lambda_{1}+\omega_{n}\left|\lambda_{3}-\lambda_{4}\right| .
$$

Proof. Define the tracking error $\widetilde{q}=z-q$. By using (11), one can obtain

$$
\dot{\tilde{q}}=-\omega_{n} \tilde{q}-\dot{q} .
$$

Consider a Lyapunov function candidate (LFC)

$$
V_{\widetilde{q}}=\widetilde{q}^{2} .
$$

Then, the time derivative of (15) is

$$
\dot{V}_{\tilde{q}} \leq-2 \omega_{n} \widetilde{q}^{2}+2|\dot{q}| \sqrt{(\widetilde{q})^{2}} \leq-2 \omega_{n} V_{\widetilde{q}}+2 \lambda_{2} \sqrt{V_{\widetilde{q}}} .
$$

Using inequality (16), one obtains

$$
|\widetilde{q}| \leq e^{-\omega_{n} t}|\widetilde{q}(0)|+\frac{\lambda_{2}\left(1-e^{-\omega_{n} t}\right)}{\omega_{n}} .
$$

Thus, for all $t \geq 0$,

$$
|\widetilde{q}| \leq\left|\lambda_{3}-\lambda_{4}\right|+\frac{\lambda_{2}}{\omega_{n}} .
$$

Because $|q(t)| \leqslant \lambda_{1}$, it follows that $z$ is bounded and satisfies

$$
\begin{aligned}
|z| & =|z-q+q| \leq|z-q|+|z| \\
& \leq \lambda_{1}+\left|\lambda_{3}-\lambda_{4}\right|+\frac{\lambda_{1}}{\omega_{n}} .
\end{aligned}
$$

From (11) and (18), one obtains

$$
\begin{aligned}
|\dot{z}| & =\omega_{n}|z-q| \leq \omega_{n}\left(\left|\lambda_{3}-\lambda_{4}\right|+\frac{\lambda_{1}}{\omega_{n}}\right) \\
& =\omega_{n}\left|\lambda_{3}-\lambda_{4}\right|+\lambda_{1} .
\end{aligned}
$$

That is, $z_{1}$ and $\dot{z}_{1}$ are bounded for all $t \geq 0$. 
Remark 10. For a given input $q$, the larger value of the FOCF parameter $\omega_{n}$ will decrease the estimated error $z-q$, which leads to better final estimate accuracy.

Remark 11. To address the problem of excessive estimation error in the initial time, we assume that the FOCF initial values are $z(0)=q(0)$ in this paper. Combined with (11) and (18), the following inequalities hold for all $t \geq 0$ :

$$
\begin{aligned}
& |z| \leq \frac{\lambda_{1}}{\omega_{n}}+\lambda_{1}, \\
& |\dot{z}| \leq \lambda_{2} .
\end{aligned}
$$

2.5. Control Formulation. This article aims to design a containment control scheme such that all the followers in the group move into the convex hull formed by the leaders [28, 40]; that is,

$$
\lim _{t \rightarrow \infty} d\left(\mathbf{p}_{i}-\operatorname{Co}\left(X_{L}\right)\right)=0
$$

where $\mathbf{p}_{i}=\left[x_{i}, y_{i}\right]^{T}$ is the position of the follower $i(i=$ $1,2, \ldots, N) ; \mathbf{X}_{L}:=\left[\phi_{M+1}^{T}, \ldots, \phi_{N}^{T}\right]^{T} ; d(x, \mathbf{M})$ denotes the distance from a point $x \in \mathbf{R}^{2}$ to a set $\mathbf{M} \in \mathbf{R}^{2}$; that is,

$$
d(x, \mathbf{M}):=\inf _{y \in M}|x-y| .
$$

Lemma 12. Under Assumption 4, all the eigenvalues of $\mathbf{L}_{1}$ have positive real parts. Furthermore, each of $-\mathbf{L}_{1}^{-1} \mathbf{L}_{2}$ is nonnegative, and all the row sums of this matrix are equal to 1 [41].

Remark 13. Let $\mathbf{X}_{L}(t):=\left[\phi_{M+1}^{T}(t), \ldots, \phi_{N}^{T}(t)\right]^{T}$ be the positions of the dynamic leaders. From Definition 2 and Assumption 4, Lemma 12 implies that every point of $-\left(\mathbf{L}_{1}^{-1} \mathbf{L}_{2} \otimes \mathbf{I}_{2}\right) \mathbf{X}_{L}(t)$ is in the convex hull spanned by the leaders $\mathbf{X}_{L}(t)$.

With this previous notation, the control problem under study can be formulated as below.

Control Objective. Consider the model given by (6) and (7) under Assumptions 3-5, and the objective of this work is to design a robust cooperative controller for each ship on the basis of its local states and the information from neighbors and a portion of the leaders such that

$$
\lim _{t \rightarrow \infty}\left\|\mathbf{p}_{i}(t)-\mathbf{p}_{d i}(t)\right\| \leq \sigma_{i}
$$

where $\sigma_{i}$ is a positive constant, $\mathbf{p}_{i}(t) \in \mathbf{R}^{2}$ denotes the actual position of follower $i$, and $\mathbf{p}_{d i}(t) \in \mathbf{R}^{2}$ denotes the desired position of follower $i$ which satisfies

$$
\mathbf{P}_{d}(t)=-\left(\mathbf{L}_{1}^{-1} \mathbf{L}_{2} \otimes \mathbf{I}_{2}\right) \mathbf{X}_{L}(t),
$$

where $\mathbf{P}_{d}(t)=\left[\mathbf{p}_{d 1}^{T}(t), \ldots, \mathbf{p}_{d i}^{T}(t), \ldots, \mathbf{p}_{d M}^{T}(t)\right]^{T}$.

Remark 14. Because of the movement of the ship affected by the ocean environment and the filtering error induced by FOCF, the tracking error $\mathbf{p}_{i}-\mathbf{p}_{d i}$ cannot converge to zero but can be uniformly ultimately bounded by $\sigma_{i}$, which will be discussed in detail in the next part.

\section{Control Design}

\subsection{Kinematic Loop Design}

Step 1. First, to prepare for the design, the following error variables are introduced:

$$
\begin{gathered}
e_{U i}^{0}=U_{i}-\boldsymbol{\alpha}_{U i}, \\
e_{\psi T i}^{0}=\psi_{T i}-\boldsymbol{\alpha}_{\psi T i},
\end{gathered}
$$

where $\boldsymbol{\alpha}_{U i}$ and $\boldsymbol{\alpha}_{\psi T i}$ denote the virtual control laws of $U_{T i}$ and $\psi_{T i}$, respectively. Then, it is convenient to expand (6) into

$$
\begin{aligned}
\dot{\mathbf{p}}_{i} & =\mathbf{V}_{i}=\boldsymbol{\alpha}_{V i}+\left(\mathbf{V}_{i}-\boldsymbol{\alpha}_{V i}\right) \\
& =\boldsymbol{\alpha}_{V i}+\boldsymbol{\Phi}_{1}\left(\psi_{T i}\right) e_{U i}^{0}+\boldsymbol{\Phi}_{2}\left(\boldsymbol{\alpha}_{\psi T i}\right) \boldsymbol{\Phi}_{3}\left(e_{\psi T i}^{0}\right) \boldsymbol{\alpha}_{U i},
\end{aligned}
$$

where

$$
\begin{aligned}
\mathbf{p}_{i} & =\left[x_{i}, y_{i}\right]^{T}, \\
\mathbf{V}_{i} & =\left[U_{T i} \cos \psi_{T i}, U_{T i} \sin \psi_{T i}\right]^{T}, \\
\boldsymbol{\alpha}_{V i} & =\left[\boldsymbol{\alpha}_{U i} \cos \boldsymbol{\alpha}_{\psi T i}, \boldsymbol{\alpha}_{U i} \sin \boldsymbol{\alpha}_{\psi T i}\right]^{T}, \\
\boldsymbol{\Phi}_{1}(\cdot) & =[\cos (\cdot), \sin (\cdot)]^{T}, \\
\boldsymbol{\Phi}_{2}(\cdot) & =\left[\begin{array}{ll}
\cos (\cdot) & -\sin (\cdot) \\
\sin (\cdot) & \cos (\cdot)
\end{array}\right], \\
\boldsymbol{\Phi}_{3}(\cdot) & =\left[\begin{array}{ll}
\cos (\cdot)-1, \sin (\cdot)
\end{array}\right]^{T} .
\end{aligned}
$$

It is obvious that $\boldsymbol{\alpha}_{V i}$ is the virtual control law for (27) which will be specified later. To solve the control problem (24), $\boldsymbol{\alpha}_{V i}$ based on its local states and the information from neighbors and a portion of the leaders is designed as below:

$$
\begin{aligned}
& \boldsymbol{\alpha}_{V i}=\frac{1}{\sum_{j=1}^{N} a_{i j}}\left[\sum_{j=1}^{M} a_{i j} \dot{\mathbf{p}}_{j}+\sum_{j=M+1}^{N} a_{i j} \dot{\boldsymbol{\phi}}_{j}-k_{c i} \sum_{j=1}^{N} a_{i j} \mathbf{p}_{j}\right. \\
& \left.+k_{c i} \sum_{j=1}^{M} a_{i j} \mathbf{p}_{j}+k_{c i} \sum_{j=M+1}^{N} a_{i j} \phi_{j}\right]
\end{aligned}
$$

where $k_{c i}>0$ is a constant. To make the presentation clearer, the following notations are used:

$$
\begin{aligned}
\mathbf{L}_{1}^{-1} & =\left[b_{i j}\right]_{M \times M}, \\
\mathbf{L}_{1}^{-1} \mathbf{L}_{2} & =\left[c_{i j}\right]_{M \times(N-M)}, \\
d_{i} & =\sum_{j=1}^{N} a_{i j}, \\
\mathbf{s}_{i}(t) & =\sum_{j=1}^{M} a_{i j}\left(\mathbf{p}_{i}-\mathbf{p}_{j}\right)+\sum_{j=M+1}^{N} a_{i j}\left(\mathbf{p}_{i}-\boldsymbol{\phi}_{j}\right) .
\end{aligned}
$$

Then, the following lemma is presented. 
Lemma 15. Consider the model described by (27) canceling the terms $\mathbf{V}_{i}-\boldsymbol{\alpha}_{V i}$, with the virtual control law (29). If the proposed control system satisfies Assumptions 4 and 5, the following properties can be given: (1) the control objective (24) is achieved; that is, $\mathbf{p}_{i}(t)-\mathbf{p}_{d i}(t)$, as $t \rightarrow \infty$, where $\mathbf{p}_{d i}(t) \in \mathbf{R}^{2}$ is defined as (25); (2) the positive constants $\rho_{1 i}$ and $\rho_{2 i}$ exist, such that $\left\|\boldsymbol{\alpha}_{V i}\right\| \leq \rho_{1 i}$ and $\left\|\dot{\boldsymbol{\alpha}}_{V i}\right\| \leq \rho_{2 i}$ for all $t \geq t_{0}$, respectively; (3) the norm $\left\|\boldsymbol{\alpha}_{V i}\right\|$ is strictly positive for all $t \geq t_{0}$ if the control parameter satisfies

$$
k_{c i}<\frac{\sum_{j=M+1}^{N}\left|c_{i j}\right| d_{2 j}}{\left(\sum_{j=1}^{M}\left|b_{i j}\right|\left\|\mathbf{s}_{j}\left(t_{0}\right)\right\|\right)},
$$

where $i=1,2, \ldots, M$.

Proof. This proof is divided into three parts.

(1) By substituting (29) into (27), canceling the term $\mathbf{V}_{i}$ $\boldsymbol{\alpha}_{V i}$, and multiplying both sides of (27) by $d_{i}$, one obtains

$$
\begin{aligned}
& \sum_{j=1}^{N} a_{i j} \dot{\mathbf{p}}_{i}-\sum_{j=1}^{M} a_{i j} \dot{\mathbf{p}}_{j}-\sum_{j=M+1}^{N} a_{i j} \dot{\boldsymbol{\phi}}_{j} \\
& =-k_{c i}\left(\sum_{j=1}^{N} a_{i j} \mathbf{p}_{i}-\sum_{j=1}^{M} a_{i j} \mathbf{p}_{j}-\sum_{j=M+1}^{N} a_{i j} \phi_{j}\right) .
\end{aligned}
$$

From (32), it follows that

$$
\begin{aligned}
& \sum_{j=1}^{M} a_{i j}\left(\dot{\mathbf{p}}_{i}-\dot{\mathbf{p}}_{j}\right)+\sum_{j=M+1}^{N} a_{i j}\left(\dot{\mathbf{p}}_{i}-\dot{\boldsymbol{\phi}}_{j}\right) \\
& \quad=-k_{c i}\left[\sum_{j=1}^{M} a_{i j}\left(\mathbf{p}_{i}-\mathbf{p}_{j}\right)+\sum_{j=M+1}^{N} a_{i j}\left(\mathbf{p}_{i}-\boldsymbol{\phi}_{j}\right)\right] .
\end{aligned}
$$

Hence, defining $\mathbf{P}(t)=\left[\mathbf{p}_{1}^{T}, \ldots, \mathbf{p}_{i}^{T}, \ldots, \mathbf{p}_{M}^{T}\right]^{T}$, (33) can be rewritten in a matrix form as below:

$$
\begin{aligned}
\left(\mathbf{L}_{1}\right. & \left.\otimes \mathbf{I}_{2}\right) \dot{\mathbf{P}}(t)+\left(\mathbf{L}_{2} \otimes \mathbf{I}_{2}\right) \dot{\mathbf{X}}_{L}(t) \\
& =-\mathbf{K}_{c}\left[\left(\mathbf{L}_{1} \otimes \mathbf{I}_{2}\right) \times \mathbf{P}(t)+\left(\mathbf{L}_{2} \otimes \mathbf{I}_{2}\right) \mathbf{X}_{L}(t)\right],
\end{aligned}
$$

where $\mathbf{I}_{2}=\operatorname{diag}(1,1)$ and $\mathbf{K}_{c}=\operatorname{diag}\left(k_{1 c}, k_{2 c}, \ldots, k_{M c}\right)$. From (34), it can be observed that $\left(\mathbf{L}_{1} \otimes \mathbf{I}_{2}\right) \mathbf{P}(t)+\left(\mathbf{L}_{2} \otimes \mathbf{I}_{2}\right) \mathbf{X}_{L}(t)$ exponentially converges to zero as $t \rightarrow \infty$. Because $\mathbf{L}_{1}^{-1}$ exists [42], $\mathbf{P}(t)$ converges to $-\left(\mathbf{L}_{1}^{-1} \mathbf{L}_{2} \otimes \mathbf{I}_{2}\right) \mathbf{X}_{L}(t)$ as $t \rightarrow \infty$; that is, the objective (24) is reached.

(2) Multiplying both sides of (34) with $\mathbf{L}_{1}^{-1} \otimes \mathbf{I}_{2}$ results in the following matrix form:

$$
\begin{gathered}
\dot{\mathbf{P}}(t)+\left(\mathbf{L}_{1}^{-1} \mathbf{L}_{2} \otimes \mathbf{I}_{2}\right) \dot{\mathbf{X}}_{L}(t)=-\mathbf{K}_{c}\left(\mathbf{L}_{1}^{-1} \otimes \mathbf{I}_{2}\right) \\
\cdot\left[\left(\mathbf{L}_{1} \otimes \mathbf{I}_{2}\right) \times \mathbf{P}(t)+\left(\mathbf{L}_{2} \otimes \mathbf{I}_{2}\right) \mathbf{X}_{L}(t)\right] .
\end{gathered}
$$

From (30) and (35), it follows that

$$
\begin{aligned}
\dot{\mathbf{p}}_{i}(t) & =\boldsymbol{\alpha}_{V i}=-\sum_{j=M+1}^{N} c_{i j} \dot{\boldsymbol{\phi}}_{j}(t)-k_{c i} \sum_{j=1}^{M} b_{i j} \mathbf{s}_{j}(t) \\
& =-\sum_{j=M+1}^{N} c_{i j} \dot{\boldsymbol{\phi}}_{j}(t)-k_{c i} \sum_{j=1}^{M} b_{i j} \mathbf{s}_{j}\left(t_{0}\right) e^{-k_{j} t} .
\end{aligned}
$$

By use of the triangle inequality, one obtains the upper bounds of the virtual control and its derivative

$$
\begin{aligned}
& \left\|\boldsymbol{\alpha}_{V i}\right\| \leq \sum_{j=M+1}^{N}\left|c_{i j}\right| d_{2 j}+k_{c i} \sum_{j=1}^{M}\left|b_{i j}\right|\left\|\mathbf{s}_{j}\left(t_{0}\right)\right\|:=\rho_{1 i} \\
& \left\|\dot{\boldsymbol{\alpha}}_{V i}\right\| \leq \sum_{j=M+1}^{N} c_{i j} b_{i j}+k_{c i} \sum_{j=1}^{M} k_{j}\left\|\mathbf{s}_{j}\left(t_{0}\right)\right\|:=\rho_{2 i},
\end{aligned}
$$

where $\rho_{1 i}$ and $\rho_{2 i}$ are positive constants. Thus, $\boldsymbol{\alpha}_{V i}$ and $\dot{\boldsymbol{\alpha}}_{V i}$ are bounded for all $t \geq 0$.

(3) Then, with the aid of the triangle inequality, the norm of $\boldsymbol{\alpha}_{V i}$ can be expressed as follows:

$$
\begin{aligned}
\left\|\boldsymbol{\alpha}_{V i}\right\| & \geq \sum_{j=M+1}^{N}\left\|c_{i j} \dot{\phi}_{i j}(t)\right\|-k_{c i} \sum_{j=1}^{M}\left\|b_{i j} \mathbf{s}_{j}(t) e^{-k_{j} t}\right\| \\
& \geq \sum_{j=M+1}^{N} c_{i j} d_{1 j}-k_{c i} \sum_{j=1}^{M}\left|b_{i j}\right|\left\|\mathbf{s}_{j}\left(t_{0}\right)\right\|:=\rho_{3 i},
\end{aligned}
$$

where $\rho_{3 i}$ is a constant. Clearly, if condition (31) holds, it would imply the strict positiveness of the norm of $\boldsymbol{\alpha}_{V i}$ for all $t \geq 0$. This calculation completes this proof.

Remark 16. To facilitate the analysis of the properties of the proposed containment control law (29), the tracking error variable $\mathbf{V}_{i}-\boldsymbol{\alpha}_{V i}$ is omitted, which will be considered in the following BP design procedure.

At this point, one should also notice that the design of this control system will utilize the virtual control of $\boldsymbol{\alpha}_{U i}$ and $\boldsymbol{\alpha}_{\psi T i}$ in the following design steps. To facilitate the computation of the virtual control, let $\boldsymbol{\alpha}_{V i}$ in (29) be expressed as

$$
\boldsymbol{\alpha}_{V i}=\left[\eta_{x i}, \eta_{y i}\right]^{T},
$$

where

$$
\begin{aligned}
\eta_{x i} & =\frac{1}{d_{i}}\left[\sum_{j=1}^{M} a_{i j} \dot{x}_{j}+\sum_{j=M+1}^{N} a_{i j} \dot{x}_{d j}-k_{c i} \sum_{j=1}^{N} a_{i j} x_{j}\right. \\
& \left.+k_{c i} \sum_{j=1}^{M} a_{i j} x_{j}+k_{c i} \sum_{j=M+1}^{N} a_{i j} x_{d j}\right], \\
\eta_{y i} & =\frac{1}{d_{i}}\left[\sum_{j=1}^{M} a_{i j} \dot{y}_{j}+\sum_{j=M+1}^{N} a_{i j} \dot{y}_{d j}-k_{c i} \sum_{j=1}^{N} a_{i j} y_{j}\right. \\
& \left.+k_{c i} \sum_{j=1}^{M} a_{i j} y_{j}+k_{c i} \sum_{j=M+1}^{N} a_{i j} y_{d j}\right] .
\end{aligned}
$$

Then, combing with (28), (29), and (39), the expressions of $\boldsymbol{\alpha}_{U i}$ and $\boldsymbol{\alpha}_{\psi T i}$ can be given by

$$
\begin{aligned}
\boldsymbol{\alpha}_{U i} & =\sqrt{\eta_{x i}^{2}+\eta_{y i}^{2}}, \\
\boldsymbol{\alpha}_{\psi T i} & =\operatorname{atan} 2\left(\eta_{y i}, \eta_{x i}\right) .
\end{aligned}
$$




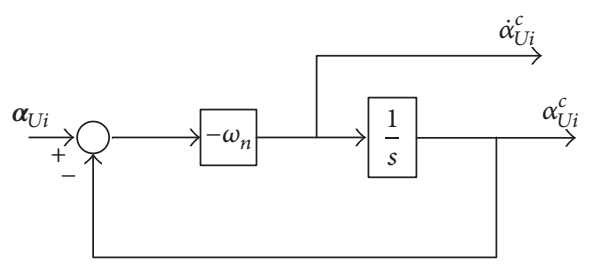

Figure 2: Configuration of FOCF.

Lemma 17. For $\boldsymbol{\alpha}_{U i}$ and $\boldsymbol{\alpha}_{\psi T i}$ described by (41), if $k_{c i}$ in (36) satisfies condition (31), then the positive constants $B_{1 i}, D_{1 i}, B_{2 i}$, and $D_{2 i}$ exist, such that

$$
\begin{gathered}
\left|\boldsymbol{\alpha}_{U i}\right| \leq B_{1 i}, \\
\left|\dot{\alpha}_{U i}\right| \leq D_{1 i} \\
\left|\boldsymbol{\alpha}_{\psi T i}\right| \leq B_{2 i}, \\
\left|\boldsymbol{\alpha}_{\psi T i}\right| \leq D_{2 i} .
\end{gathered}
$$

Proof. From (28), $\boldsymbol{\alpha}_{U i}$ is bounded by

$$
\left|\boldsymbol{\alpha}_{U i}\right|=\left\|\boldsymbol{\alpha}_{V i}\right\| \leq \rho_{1 i}:=B_{1 i}
$$

Differentiating both sides of the first equation of (41) gives

$$
\dot{\alpha}_{U i}=\frac{\boldsymbol{\alpha}_{V i}^{T} \dot{\alpha}_{V i}}{\left(2 \alpha_{U i}\right)} \text {. }
$$

By use of Lemma 9, one can obtain

$$
\left|\dot{\alpha}_{U i}\right| \leq 0.25\left(1+\left\|\dot{\boldsymbol{\alpha}}_{V i}\right\|\right) \leq 0.25\left(1+\rho_{2 i}\right)=: D_{1 i} .
$$

According to the expression of $\boldsymbol{\alpha}_{\psi T i}$ in (41), $\boldsymbol{\alpha}_{\psi T i}$ is bounded by

$$
\left|\boldsymbol{\alpha}_{\psi T i}\right| \leq \pi:=B_{2 i} .
$$

As inequality (45), $\dot{\alpha}_{\psi T i}$ satisfies the following:

$$
\left|\dot{\alpha}_{\psi T i}\right| \leq \frac{\left\|\dot{\boldsymbol{\alpha}}_{V i}\right\|}{\left|\boldsymbol{\alpha}_{U i}\right|} \leq \frac{\rho_{2 i}}{\rho_{3 i}}:=D_{2 i} .
$$

This calculation completes this proof.

Step 2. In this work, the BP method is applied to the strictfeedback nonlinear system of (6) and (7). However, it uses the derivatives of the virtual control such as $\boldsymbol{\alpha}_{U i}$ and $\boldsymbol{\alpha}_{\psi T i}$, which increases the complexity of calculations and needs the acceleration information from its neighbors. To avoid the problems described previously, FOCF is introduced to estimate each virtual control law and its derivative $[30,43]$.

To facilitate the expressions of the variables involved in filtered BP method, a particular notation is introduced in this paper. For example, let $\boldsymbol{\alpha}_{U i}$ go through FOCF depicted in Figure 2 to obtain $\alpha_{U i}^{c}$ and $\dot{\alpha}_{U i}^{c}$, which represent the estimated values of $\boldsymbol{\alpha}_{U i}$ and $\dot{\alpha}_{U i}$, respectively. Similarly, we can also define the variables $\alpha_{\psi T i}^{c}, \dot{\alpha}_{\psi T i}^{c}, \alpha_{r i}^{c}$, and $\dot{\alpha}_{r i}^{c}$. For the sake of the design work, the following tracking errors are defined as

$$
\begin{gathered}
e_{U i}=U_{i}-\alpha_{U i}^{c}, \\
e_{\psi T i}=\psi_{T i}-\alpha_{\psi T i}^{c}, \\
e_{r i}=r_{i}-\alpha_{r i}^{c} .
\end{gathered}
$$

Remark 18. It is noted the boundedness of $\boldsymbol{\alpha}_{U i}, \dot{\alpha}_{U i}, \boldsymbol{\alpha}_{\psi T i}$, and $\dot{\alpha}_{\psi T i}$ ensures the boundedness of $\alpha_{U i}^{c}, \dot{\alpha}_{U i}^{c} \dot{\alpha}_{U i}^{c}$, and $\dot{\alpha}_{\psi T i}^{c}$ by Lemma 9. According to Lemma 17, $\boldsymbol{\alpha}_{U i} \dot{\alpha}_{U i}, \boldsymbol{\alpha}_{\psi T i}$, and $\dot{\alpha}_{\psi T i}$ are bounded by $B_{1 i}, D_{1 i}, B_{2 i}$, and $D_{2 i}$, respectively, and then the constants $B_{3 i}, D_{3 i}, B_{4 i}$, and $B_{4 i}$ exist and satisfy

$$
\begin{gathered}
\left|\alpha_{U i}^{c}\right| \leq B_{3 i}, \\
\left|\dot{\alpha}_{U i}^{c}\right| \leq D_{3 i}, \\
\left|\alpha_{\psi T i}^{c}\right| \leq B_{4 i}, \\
\left|\dot{\alpha}_{\psi T i}^{c}\right| \leq D_{4 i} .
\end{gathered}
$$

With (48) applied, the kinematic model (6) can be rewritten as

$$
\begin{aligned}
\dot{\mathbf{p}}_{i}= & \mathbf{V}_{i}=\boldsymbol{\alpha}_{V i}+\left(\mathbf{V}_{i}-\mathbf{V}_{i}^{c}\right)+\left(\mathbf{V}_{i}^{c}-\boldsymbol{\alpha}_{V i}\right) \\
= & \boldsymbol{\alpha}_{V i}+\boldsymbol{\Phi}_{1}\left(\psi_{T i}\right) e_{U i}+\boldsymbol{\Phi}_{2}\left(\alpha_{\psi T i}^{c}\right) \boldsymbol{\Phi}_{3}\left(e_{\psi i}\right) \alpha_{U i}^{c} \\
& +\left(\mathbf{V}_{i}^{c}-\boldsymbol{\alpha}_{V i}\right),
\end{aligned}
$$

where $\mathbf{p}_{i}, \mathbf{V}_{i}, \boldsymbol{\alpha}_{V i}, \boldsymbol{\Phi}_{1}(\cdot), \boldsymbol{\Phi}_{2}(\cdot)$, and $\boldsymbol{\Phi}_{3}(\cdot)$ are defined as (28), $\mathbf{V}_{i}^{c}=\left[\alpha_{U i}^{c} \cos \alpha_{\psi T i}^{c}, \alpha_{U i}^{c} \sin \alpha_{\psi T i}^{c}\right]^{T}$. Then, substituting (29) into (50) and multiplying both sides of (50) with $d_{i}$ give

$$
\begin{aligned}
\dot{\mathbf{s}}_{i}= & -k_{c i} \mathbf{s}_{i}+\left(\sum_{j=1}^{N} a_{i j}\right) \\
& \cdot\left[\boldsymbol{\Phi}_{1}\left(\psi_{T i}\right) e_{U i}+\boldsymbol{\Phi}_{2}\left(\alpha_{\psi T i}^{c}\right) \boldsymbol{\Phi}_{3}\left(e_{\psi T i}\right) \times \alpha_{U i}^{c}\right] \\
& +\left(\sum_{j=1}^{N} a_{i j}\right)\left(\mathbf{V}_{i}^{c}-\boldsymbol{\alpha}_{V i}\right) .
\end{aligned}
$$

Clearly, if the last two terms converge to zero, $\mathbf{s}_{i}$ exponentially converges to zero. To prepare for the following step, define the compensating tracking error vector as

$$
\begin{gathered}
\mathbf{E}_{s i}=\mathbf{s}_{i}-\xi_{s i}, \\
E_{U i}=e_{U i}-\xi_{U i}, \\
E_{\psi T i}=e_{\psi T i}-\xi_{\psi T i}, \\
E_{r i}=e_{r i}-\xi_{r i},
\end{gathered}
$$

where $\xi_{U i}, \xi_{\psi T i}$, and $\xi_{r i}$ denote the compensating signals of $e_{U i}, e_{\psi T i}$, and $e_{r i}$, respectively. In order to remove the effect of 
the nonlinear term $\mathbf{V}_{i}^{c}-\boldsymbol{\alpha}_{V i}$ in (51), a compensating signal is selected as

$$
\begin{aligned}
\dot{\boldsymbol{\xi}}_{s i} & =-k_{c i} \boldsymbol{\xi}_{s i}+\left(\sum_{j=1}^{N} a_{i j}\right)\left[\boldsymbol{\Phi}_{1}\left(\psi_{T i}\right) \boldsymbol{\xi}_{U i}\right. \\
& \left.+\boldsymbol{\Phi}_{2}\left(\boldsymbol{\alpha}_{\psi T i}\right) \frac{\boldsymbol{\Phi}_{3}\left(e_{\psi T i}\right)}{e_{\psi T i}} \times \boldsymbol{\alpha}_{U i} \boldsymbol{\xi}_{\psi T i}\right]+\left(\sum_{j=1}^{N} a_{i j}\right)\left(\mathbf{V}_{i}^{c}\right. \\
& \left.-\boldsymbol{\alpha}_{V i}\right)
\end{aligned}
$$

where the initial conditions are $\xi_{s i}=[0 ; 0]$ and $\xi_{U i}=$ $b_{0} \exp \left(-k_{U i} t\right)$. From (51), (52), and (53), one obtains

$$
\begin{aligned}
\dot{\mathbf{E}}_{s i} & =-k_{c i} \mathbf{E}_{s i}+\left(\sum_{j=1}^{N} a_{i j}\right)\left[\boldsymbol{\Phi}_{1}\left(\psi_{T i}\right) E_{U i}+\boldsymbol{\Phi}_{2}\left(\boldsymbol{\alpha}_{\psi T i}\right)\right. \\
& \left.\times \frac{\boldsymbol{\Phi}_{3}\left(e_{\psi T i}\right)}{e_{\psi T i}} \boldsymbol{\alpha}_{U i} E_{\psi T i}\right],
\end{aligned}
$$

noting that the last nonlinear term of (54) will be compensated in the next step. Then, considering the first LFC

$$
V_{1}=0.5 \sum_{i=1}^{M} \mathbf{E}_{s i}^{T} \mathbf{E}_{s i} .
$$

Taking the time derivative of $V_{1}$ along the solution of (54) gives

$$
\begin{aligned}
\dot{V}_{1}= & -k_{c i} \sum_{i=1}^{M}\left\|\mathbf{E}_{s i}\right\|^{2}+\sum_{i=1}^{M}\left(\sum_{j=1}^{M} a_{i j}\right) \mathbf{E}_{s i}^{T} \boldsymbol{\Phi}_{1}\left(\psi_{T i}\right) E_{U i} \\
& +\sum_{i=1}^{M}\left(\sum_{j=1}^{M} a_{i j}\right) \mathbf{E}_{s i}^{T} \boldsymbol{\Phi}_{2}\left(\boldsymbol{\alpha}_{\psi T i}\right) \frac{\boldsymbol{\Phi}_{3}\left(e_{\psi T i}\right)}{e_{\psi T i}} \boldsymbol{\alpha}_{U i} E_{\psi T i} .
\end{aligned}
$$

Step 3. At this step, $r_{i}$ will be considered as a control input to stabilize the tracking error $e_{U i}$. With notation (48), the kinematic model for $\psi_{T i}$ can be rewritten as below:

$$
\dot{\psi}_{T i}=r_{i}+\dot{\beta}_{i}=\alpha_{r i}+e_{r i}+\alpha_{r i}^{c}-\alpha_{r i}+\dot{\beta}_{i}
$$

where $\alpha_{r i}$ is the virtual control for $r_{i}$. To stabilize the tracking error $e_{\psi T i}$ in (48) and to compensate the last term for (56), the virtual control law $\alpha_{r i}$ is designed as

$$
\begin{aligned}
\alpha_{r i}= & \dot{\alpha}_{\psi T i}^{c}-k_{\psi T i} e_{\psi T i} \\
& -\left(\sum_{j=1}^{N} a_{i j}\right) \mathbf{E}_{s i}^{T} \Phi_{2}\left(\boldsymbol{\alpha}_{\psi T i}\right) \frac{\boldsymbol{\Phi}_{3}\left(e_{\psi T i}\right)}{e_{\psi T i}} \times \boldsymbol{\alpha}_{U i}-\dot{\beta}_{i},
\end{aligned}
$$

where $k_{\psi T i}>0$ is a constant. Then, from (34) and (35), it follows that

$$
\begin{aligned}
\dot{e}_{\psi T i}= & -k_{\psi T i} e_{\psi T i}-\left(\sum_{j=1}^{N} a_{i j}\right) \mathbf{E}_{s i}^{T} \Phi_{2}\left(\boldsymbol{\alpha}_{\psi T i}\right) \frac{\boldsymbol{\Phi}_{3}\left(e_{\psi T i}\right)}{e_{\psi T i}} \\
& \times \boldsymbol{\alpha}_{U i}+e_{r i}+\left(\alpha_{r i}^{c}-\alpha_{r i}\right) .
\end{aligned}
$$

Select the compensating signal for $e_{\psi T i}$ as

$$
\dot{\xi}_{\psi T i}=-k_{\psi T i} \xi_{\psi T i}+\xi_{r i}+\left(\alpha_{r i}^{c}-\alpha_{r i}\right)
$$

where $\xi_{\psi T i}(0)=0$. By the definition of $E_{\psi T i}$ in (52) and (59) and (60), the compensating tracking error $E_{\psi T i}$ can be expressed as

$$
\begin{aligned}
\dot{E}_{\psi T i}= & -k_{\psi T i} E_{\psi T i} \\
& -\left(\sum_{j=1}^{N} a_{i j}\right) \mathbf{E}_{s i}^{T} \boldsymbol{\Phi}_{2}\left(\boldsymbol{\alpha}_{\psi T i}\right) \frac{\boldsymbol{\Phi}_{3}\left(e_{\psi T i}\right)}{e_{\psi T i}} \boldsymbol{\alpha}_{U i}+E_{r i} .
\end{aligned}
$$

Consider the second LFC:

$$
V_{2}=V_{1}+0.5 \sum_{i=1}^{M} E_{\psi T i}^{2}
$$

whose time derivative along (56) and (61) is

$$
\begin{aligned}
\dot{V}_{2}= & \dot{V}_{1}+\sum_{i=1}^{M} E_{\psi T i} \dot{E}_{\psi T i} \\
= & -\sum_{i=1}^{M} k_{i}\left\|\mathbf{E}_{s i}\right\|^{2}-\sum_{i=1}^{M} k_{\psi T i} E_{\psi T i}^{2}+\sum_{i=1}^{M}\left(\sum_{j=1}^{N} a_{i j}\right) \mathbf{E}_{s i}^{T} \\
& \times \Phi_{1}\left(\psi_{T i}\right) E_{U i}-\sum_{i=1}^{M} E_{\psi T i} E_{r i} .
\end{aligned}
$$

It should be noted that the last two terms of (63) will be compensated in the dynamic design.

3.2. Dynamic Loop Design. This section aims to design the robust control inputs $F_{u i}$ and $F_{r i}$ which make the compensated tracking errors $E_{u i}$ and $E_{r i}$ asymptotically stable.

Step 1 (surge control design). The definition of $E_{r i}$ in (52) is chosen as the sliding surface:

$$
E_{r i}=e_{r i}-\xi_{r i} .
$$

Differentiating both sides of (64) along (7), one can obtain $E_{r i}$ dynamics as

$$
\begin{aligned}
\dot{E}_{r i} & =\dot{e}_{r i}-\dot{\xi}_{r i}=\dot{r}_{i}-\dot{\alpha}_{r i}^{c}-\dot{\xi}_{r i}=\frac{1}{m_{33 i}}\left[m_{11 i} u_{i} v_{i}\right. \\
& -m_{22 i} u_{i} v_{i}-d_{r i} r_{i}-d_{r 2 i}\left|r_{i}\right| r_{i}-d_{v r i} r_{i}+\tau_{w r i}+F_{r i} \\
& \left.-m_{33 i} \dot{\alpha}_{r i}^{c}-m_{33 i} \dot{\xi}_{r i}\right] .
\end{aligned}
$$

To ensure that $E_{r i}$ is always equal to zero, the equivalent control input $F_{r e q i}$ can be obtained by solving $\dot{E}_{r i}=0$ without the parameter uncertainties and external disturbances. Thus, $F_{\text {reqi }}$ can be chosen as

$$
\begin{aligned}
F_{r e q i}= & -\widehat{m}_{11 i} u_{i} v_{i}+\widehat{m}_{22 i} u_{i} v_{i}+\widehat{d}_{r i} r_{i}+\widehat{d}_{r 2 i}\left|r_{i}\right| r_{i} \\
& +\widehat{d}_{v r i} r_{i}+\widehat{m}_{33 i} \dot{\alpha}_{r i}^{c}+\widehat{m}_{33 i} \dot{\xi}_{r i}
\end{aligned}
$$


where “へ” denotes the estimated ship parameters. It could be easily found that the equivalent control input cannot guarantee $E_{r i}=0$ when the EDPU are considered; to eliminate the effect of the uncertainties of the control system and to ensure the convergence of $E_{r i}$ to zero, an additional control input $F_{\text {radi }}$ is introduced:

$$
F_{\text {radi }}=-q_{2 i} E_{r i}-\eta_{r i} \operatorname{sign}\left(E_{r i}\right),
$$

where $q_{2 i}$ is a positive constant and $\eta_{u i}$ is the control parameter, determined from the following bounds of the ship parameters in (7):

$$
\begin{gathered}
\left|m_{11 i}-\widehat{m}_{11 i}\right| \leq M_{11}, \\
\left|m_{22 i}-\widehat{m}_{22 i}\right| \leq M_{22}, \\
\left|d_{r i}-\widehat{d}_{r i}\right| \leq D_{r}, \\
\left|d_{r 2 i}-\widehat{d}_{r 2 i}\right| \leq D_{r 2}, \\
\left|d_{v r i}-\widehat{d}_{v r i}\right| \leq D_{v r} .
\end{gathered}
$$

Moreover, to compensate for the last term of (63), a compensated control input $F_{r c p i}$ is set to be

$$
F_{r c p i}=-E_{\psi T i} \text {. }
$$

Combined with the previously mentioned control input, the actual yaw control input $F_{r i}$ can be chosen as

$$
F_{r i}=F_{r e q i}+F_{r a d i}+F_{r c p i} .
$$

To determine $\eta_{u i}$, consider the LFC for $E_{r i}$ as

$$
V_{r}=0.5 \sum_{i=1}^{M} m_{33 i} E_{r i}^{2}
$$

whose time derivative along (65) and (70) is

$$
\begin{aligned}
\dot{V}_{r} & =\sum_{i=1}^{M} m_{33 i} E_{r i} \dot{E}_{r i}=\sum_{i=1}^{M} E_{r i}\left[\left(m_{11 i}-\widehat{m}_{11 i}\right) u_{i} v_{i}\right. \\
& +\left(\widehat{m}_{22 i}-m_{22 i}\right) u_{i} v_{i}+\left(\widehat{d}_{r i}-d_{r i}\right) r_{i} \\
& +\left(\widehat{d}_{r 2 i}-d_{r 2 i}\right)\left|r_{i}\right| r_{i}+\left(\widehat{d}_{v r i}-d_{v r i}\right) r_{i}+\tau_{w r i} \\
& +\left(\widehat{m}_{33 i}-m_{33 i}\right)\left(\dot{\alpha}_{r i}^{c}+\dot{\xi}_{r i}\right)-\eta_{r i} \operatorname{sign}\left(E_{r i}\right)-q_{2 i} E_{r i} \\
& \left.-E_{\psi T i}\right] \leq \sum_{i=1}^{M}\left|E_{r i}\right|\left[\left(M_{11}+M_{22}\right)\left|u_{i} v_{i}\right|+D_{r}\left|r_{i}\right|\right. \\
& \left.+D_{r 2} r_{i}^{2}+D_{v r}\left|r_{i}\right| M_{33}\left(\left|\dot{\alpha}_{r i}^{c}\right|+\left|\dot{\xi}_{r i}\right|\right)+\bar{\tau}_{w r i}-\eta_{r i}\right] \\
& -\sum_{i=1}^{M}\left[q_{2 i} E_{r i}^{2}+E_{r i} E_{\psi T i}\right] .
\end{aligned}
$$

Then, a reasonable choice for $\eta_{u i}$ can be given as

$$
\begin{aligned}
\eta_{r i}= & M_{11}\left|u_{i} v_{i}\right|+M_{22}\left|u_{i} v_{i}\right|+D_{r}\left|r_{i}\right|+D_{r 2} r_{i}^{2} \\
& +D_{v r}\left|r_{i}\right|+\bar{\tau}_{w r}+M_{33}\left(\left|\dot{\alpha}_{r i}^{c}\right|+\left|\dot{\xi}_{r i}\right|\right) .
\end{aligned}
$$

Considering the third LFC $V_{3}=V_{2}+V_{r}$ and taking the time derivative of $V_{3}$ along (63) give

$$
\begin{aligned}
\dot{V}_{3}= & \dot{V}_{2}+\dot{V}_{r} \\
\leq & -\sum_{i=1}^{M} k_{i}\left\|\mathbf{E}_{s i}\right\|^{2}-\sum_{i=1}^{M} k_{\psi T i} E_{\psi T i}^{2}-\sum_{i=1}^{M} q_{2 i} E_{r i}^{2} \\
& +\sum_{i=1}^{M}\left(\sum_{j=1}^{N} a_{i j}\right) \mathbf{E}_{s i}^{T} \Phi_{1}\left(\psi_{T i}\right) E_{U i} .
\end{aligned}
$$

Step 2 (yaw control design). Similar to Step 1, a sliding surface for $E_{U i}$ is introduced:

$$
E_{U i}=e_{U i}-\xi_{U i}
$$

Taking the time derivative of $E_{U i}$ along (7) results in

$$
\begin{aligned}
\dot{E}_{U i}= & \dot{e}_{U i}-\dot{\xi}_{U i}=\dot{U}_{T i}-\dot{\alpha}_{U i}^{c}-\dot{\xi}_{U i} \\
= & \frac{\cos \beta_{i}}{m_{11 i}}\left(m_{22 i} v_{i} r_{i}-d_{u i} u_{i}-d_{u 2 i}\left|u_{i}\right| u_{i}\right) \\
& +\frac{\cos \beta_{i}}{m_{11 i}} \tau_{w u i} \\
& +\frac{\sin \beta_{i}}{m_{22 i}}\left(-m_{11 i} u_{i} r_{i}-d_{v i} v_{i}-d_{v 2 i}\left|v_{i}\right| v_{i}-d_{u r i} r_{i}\right) \\
& +\frac{\sin \beta_{i}}{m_{22 i}} \tau_{w v i}+\frac{\cos \beta_{i}}{m_{11 i}} F_{u i}-\dot{\alpha}_{U i}^{c}-\dot{\xi}_{U i} .
\end{aligned}
$$

Similar to the previous step, the equivalent control input $F_{\text {ueqi }}$ can be designed as

$$
\begin{aligned}
F_{u e q i} & =-\widehat{m}_{22 i} v_{i} r_{i}+\widehat{d}_{u i} u_{i}+\widehat{d}_{u 2 i}\left|u_{i}\right| u_{i} \\
& +\frac{\sin \beta_{i}}{\cos \beta_{i}}\left(\frac{\widehat{m}_{11 i}^{2}}{\widehat{m}_{22 i}} u_{i} r_{i}+\frac{\widehat{m}_{11 i} \widehat{d}_{v i}}{\widehat{m}_{22 i}} v_{i}+\frac{\widehat{m}_{11 i} \widehat{d}_{v 2 i}}{\widehat{m}_{22 i}}\left|v_{i}\right| v_{i}\right. \\
& \left.+\frac{\widehat{m}_{11 i} \widehat{d}_{v r i}}{\widehat{m}_{22 i}} r_{i}\right)+\frac{1}{\cos \beta_{i}} \widehat{m}_{11 i}\left(\dot{\alpha}_{U i}^{c}+\dot{\xi}_{U i}\right),
\end{aligned}
$$

where “へ” denotes the estimated ship parameters. To compensate for the last term of inequality (74) and reject the uncertainties, the actual surge control input can be chosen as

$$
\begin{aligned}
& F_{u i}=F_{u e q i}+F_{u a d i}, \\
& F_{u a d i}=-\frac{1}{\cos \beta_{i}}\left[\left(\sum_{j=1}^{N} a_{i j}\right) \mathbf{E}_{s i}^{T} \Phi_{1}\left(\psi_{T i}\right)+q_{1 i} E_{u i}\right. \\
& \left.\quad+\eta_{u i} \operatorname{sign}\left(E_{u i}\right)\right],
\end{aligned}
$$


where $q_{1 i}>0$ is a constant; $\eta_{u i}$ can be determined by the following bounds of the ship parameters in (7):

$$
\begin{aligned}
&\left|d_{u i}-\widehat{d}_{u i}\right| \leq D_{u}, \\
&\left|d_{u 2 i}-\widehat{d}_{u 2 i}\right| \leq D_{u 2}, \\
&\left|m_{33 i}-\widehat{m}_{33 i}\right| \leq M_{33}, \\
&\left|d_{v i}-\widehat{d}_{v i}\right| \leq D_{v}, \\
&\left|d_{v 2 i}-\widehat{d}_{v 2 i}\right| \leq D_{v 2}, \\
&\left|\frac{m_{11 i}}{m_{22 i}}\right| \leq H_{0}, \\
&\left|\frac{m_{11 i}^{2}}{m_{22 i}}-\frac{\widehat{m}_{11 i}^{2}}{\widehat{m}_{22 i}}\right| \leq H_{u r}, \\
&\left|\frac{m_{11 i} d_{v i}}{m_{22 i}}-\frac{\widehat{m}_{11 i} \widehat{d}_{v i}}{\widehat{m}_{22 i}}\right| \leq H_{v}, \\
&\left|\frac{m_{11 i} d_{v 2 i}}{m_{22 i}}-\frac{\widehat{m}_{11 i} \widehat{d}_{v 2 i}}{\widehat{m}_{22 i}}\right| \leq H_{v 2}, \\
&\left|\frac{m_{11 i} d_{v r i}}{m_{22 i}}-\frac{\widehat{m}_{11 i} \widehat{d}_{v r i}}{\widehat{m}_{22 i}}\right| \leq H_{r},
\end{aligned}
$$

and, to determine $\eta_{u i}$, we consider the LFC for $E_{U i}$ as

$$
V_{U}=0.5 \sum_{i=1}^{M} m_{11 i} E_{U i}^{2}
$$

whose time derivative along (76) and (78) is

$$
\begin{aligned}
\dot{V}_{U} & =\sum_{i=1}^{M} E_{U i}\left\{\operatorname { c o s } \beta _ { i } \left[\left(m_{22 i}-\widehat{m}_{22 i}\right) v_{i} r_{i}\right.\right. \\
& \left.+\left(\widehat{d}_{u i}-d_{u i}\right) u_{i}+\left(\widehat{d}_{u 2 i}-d_{u 2 i}\right)\left|u_{i}\right| u_{i}\right]+\cos \beta_{i} \tau_{w u i} \\
& +\sin \beta_{i} \frac{m_{11 i}}{m_{22 i}} \tau_{w v i}+\sin \beta_{i}\left[\left(\frac{\widehat{m}_{11 i}^{2}}{\widehat{m}_{22 i}}-\frac{m_{11 i}^{2}}{m_{22 i}}\right) u_{i} r_{i}\right. \\
& +\left(\frac{\widehat{m}_{11 i} \widehat{d}_{v i}}{\widehat{m}_{22 i}}-\frac{m_{11 i} d_{v i}}{m_{22 i}}\right) v_{i} \\
& +\left(\frac{\widehat{m}_{11 i} \widehat{d}_{v 2 i}}{\widehat{m}_{22 i}}-\frac{m_{11 i} d_{v 2 i}}{m_{22 i}}\right)\left|v_{i}\right| v_{i} \\
& \left.+\left(\frac{\widehat{m}_{11 i} \widehat{d}_{v 2 i}}{\widehat{m}_{22 i}}-\frac{m_{11 i} d_{v 2 i}}{m_{22 i}}\right) r_{i}\right]+\left(\widehat{m}_{11 i}-m_{11 i}\right)\left(\dot{\alpha}_{U i}\right. \\
& \left.\left.+\dot{\xi}_{U i}\right)-\eta_{u i} \operatorname{sign}\left(E_{u i}\right)\right\}-\sum_{i=1}^{M} q_{1 i} E_{u i}^{2}-\sum_{i=1}^{M}\left(\sum_{j=1}^{M} a_{i j}\right) \\
& \cdot \mathbf{E}_{s i}^{T} \boldsymbol{\Phi}_{1}\left(\psi_{T i}\right) E_{U i} .
\end{aligned}
$$

According to (80) and (82), computing the first-order derivative of $V_{U}$ results in

$$
\begin{aligned}
\dot{V}_{U} & \leq \sum_{i=1}^{M}\left|E_{U i}\right|\left\{M_{11}\left|v_{i} r_{i}\right|+D_{u}\left|u_{i}\right|+D_{u 2} u_{i}^{2}+\bar{\tau}_{w u i}\right. \\
& +H_{0} \bar{\tau}_{w v i}+H_{u r}\left|u_{i} r_{i}\right|+H_{v}\left|v_{i}\right|+H_{v 2} v_{i}^{2}+H_{r}\left|r_{i}\right| \\
& \left.+M_{11}\left(\left|\dot{\alpha}_{U i}\right|+\left|\dot{\xi}_{U i}\right|\right)-\eta_{u i}\right\}-\sum_{i=1}^{M} q_{1 i} E_{U i}^{2} \\
& -\sum_{i=1}^{M}\left(\sum_{j=1}^{M} a_{i j}\right) \mathbf{E}_{s i}^{T} \Phi_{1}\left(\psi_{T i}\right) E_{U i} .
\end{aligned}
$$

To stabilize $E_{U i}$ at the origin, $\eta_{u i}$ can be defined as

$$
\begin{aligned}
\eta_{u i}= & M_{11}\left|v_{i} r_{i}\right|+D_{u}\left|u_{i}\right|+D_{u 2} u_{i}^{2}+H_{u r}\left|u_{i} r_{i}\right| \\
& +H_{v}\left|v_{i}\right|+H_{v 2} v_{i}^{2}+H_{r}\left|r_{i}\right|+\bar{\tau}_{w u i}+H_{0} \bar{\tau}_{w v i} \\
& +M_{11}\left(\left|\dot{\alpha}_{U i}\right|+\left|\dot{\xi}_{U i}\right|\right) .
\end{aligned}
$$

Substituting (84) into (83), one obtains

$$
\dot{V}_{U} \leq-\sum_{i=1}^{M} q_{1 i} E_{U i}^{2}-\sum_{i=1}^{M}\left(\sum_{j=1}^{N} a_{i j}\right) \mathbf{E}_{s i}^{T} \boldsymbol{\Phi}_{1}\left(\psi_{T i}\right) E_{U i} .
$$

Consider the overall LFC $V_{4}=V_{3}+V_{U}$; take the time derivative of $V_{4}$ along (74) and (85) gives

$$
\begin{aligned}
\dot{V}_{4} \leq & \dot{V}_{3}+\dot{V}_{U} \\
\leq & -\sum_{i=1}^{M} k_{i}\left\|\mathbf{E}_{s i}\right\|^{2}-\sum_{i=1}^{M} k_{\psi T i} E_{\psi T i}^{2}-\sum_{i=1}^{M} q_{2 i} E_{r i}^{2} \\
& -\sum_{i=1}^{M} q_{1 i} E_{U i}^{2} .
\end{aligned}
$$

3.3. Stability Analysis of Closed-Loop System. This section consists of four steps.

Step 1 (boundedness of $\mathbf{E}_{s i}$ and $E_{U i}$ ). By using equalities (68) and (80), (86) can be derived as

$$
\begin{aligned}
\dot{V}_{4} \leq & -\sum_{i=1}^{M} k_{c i}\left\|\mathbf{E}_{s i}\right\|^{2}-\sum_{i=1}^{M} k_{\psi T i} E_{\psi i}^{2} \\
& -\sum_{i=1}^{M} \frac{q_{2 i}}{\widehat{m}_{33 i}+M_{33}} m_{33 i} E_{r i}^{2} \\
& -\sum_{i=1}^{M} \frac{q_{1 i}}{\widehat{m}_{11 i}+M_{11}} m_{11 i} E_{U i}^{2} \leq-\rho V_{4},
\end{aligned}
$$

where $\rho=\min \left\{2 k_{c i}, 2 k_{\psi i}, 2 q_{2 i} /\left(\widehat{m}_{33}+M_{33}\right), 2 q_{1 i} /\left(\widehat{m}_{11}+M_{11}\right)\right\}$.

Then, one can obtain

$$
0.5 \sum_{i=1}^{M}\left\|\mathbf{E}_{s i}\right\|^{2} \leq V_{4}(t) \leq V_{4}(0) e^{-\rho t}
$$


From equality (88), one can conclude that $\left|E_{U}(t)\right|,\left\|E_{s i}(t)\right\| \leq$ $\chi$ (where $\left.\chi=2 \sqrt{V_{4}(0)}\right)$ for all $t \geq 0$.

Step 2 (boundedness of $\beta_{i}$ ). The definition of $E_{U i}$ in (52) and the definition of $e_{U i}$ in (48) can be rewritten as

$$
E_{U i}=U_{i}-\alpha_{U i}^{c}-\zeta_{U i} .
$$

Then, the bounds of $E_{U i}, \alpha_{U i}^{c}$, and $\zeta_{U i}$ give

$$
U_{i}-B_{3 i}-b_{0} \geq-\chi .
$$

It is obviously concluded that the strict positiveness of $U_{i}$ can be guaranteed if $\zeta_{U i}$ satisfies the condition

$$
b_{0}>\chi
$$

Thus, $\left|\beta_{i}\right|<0.5 \pi$; that is, $\cos \beta_{i} \neq 0$, for all $t>0$. Because the determinant of $F_{u i}$ in (78) is given by the term $\cos \beta_{i}$, condition (91) can guarantee that $F_{u i}$ are well defined for all $t>0$.

Remark 19. Notice that, with $\beta_{i}$ being bounded by $0.5 \pi$, the constants $l_{1 i}$ and $l_{2 i}$ exist, such that $\left|\dot{\beta}_{i}\right| \leq l_{1 i},\left|\ddot{\beta}_{i}\right| \leq l_{2 i}$ all the time.

Step 3 (boundedness of $\boldsymbol{\xi}_{s i}$ ). Prior to analyzing the boundedness $\xi_{s i}$ the boundedness of $\alpha_{r i}, \alpha_{r i}^{c}$ should be discussed first. From the definition of $\alpha_{r i}$ in (58) and the Young equality, one obtains

$$
\begin{aligned}
\left|\alpha_{r i}\right| \leq & \left|\dot{\alpha}_{\psi T i}^{c}\right|+k_{\psi T i}\left|e_{\psi T i}\right| \\
& +\frac{1}{2}\left(\sum_{j=1}^{N} a_{i j}\right)\left(\left\|\mathbf{E}_{s i}\right\|^{2}+\left|\boldsymbol{\alpha}_{U i}\right|^{2}\right)+\left|\dot{\beta}_{i}\right| \\
\leq & D_{4 i}+k_{\psi T i}\left(0.5 \pi+B_{4 i}\right) \\
& +\frac{1}{2}\left(\sum_{j=1}^{N} a_{i j}\right)\left(\chi^{2}+B_{1 i}^{2}\right)+l_{1 i}^{2}=: B_{5 i}
\end{aligned}
$$

which implies the constant $D_{5 i}>0$ exists which satisfies $\left|\dot{\alpha}_{r i}\right| \leq D_{5 i}$. Combining with Lemma 9, it is seen that $\mid \alpha_{r i}-$ $\alpha_{r i}^{c} \mid \leq B_{6 i}$, where $B_{5 i}>0$ is a constant. Then, consider the LFC as

$$
\begin{aligned}
\dot{V}_{\xi}= & \sum_{i=1}^{M}\left(\xi_{s i}^{T} \dot{\xi}_{s i}+\xi_{U i} \dot{\xi}_{U i}+\xi_{\psi T i} \dot{\xi}_{\psi T i}\right) \\
\leq & -\sum_{i=1}^{M} k_{c i}\left\|\xi_{s i}\right\|^{2} \\
& +\sum_{i=1}^{M}\left(\sum_{j=1}^{N} a_{i j}\right)\left(\frac{3}{2}\left\|\xi_{s i}\right\|^{2}\right)+\sum_{i=1}^{M}\left(\sum_{j=1}^{N} a_{i j}\right) \frac{1}{2} \xi_{U i}^{2} \\
& +\sum_{i=1}^{M}\left(\sum_{j=1}^{N} a_{i j}\right) \frac{1}{2} \alpha_{U i}^{2} \xi_{\psi T i}^{2}
\end{aligned}
$$

$$
\begin{aligned}
& \left.\quad+\sum_{i=1}^{M}\left(\sum_{j=1}^{N} a_{i j}\right) \frac{1}{2}\left(\left\|\mathbf{V}_{i}^{c}\right\|^{2}+\left\|\boldsymbol{\alpha}_{V i}\right\|^{2}\right)\right)-\sum_{i=1}^{M} k_{U i} \xi_{U i}^{2} \\
& \quad-\sum_{i=1}^{M} k_{\psi T i} \xi_{\psi T i}^{2}+\sum_{i=1}^{M} \frac{1}{2}\left[\xi_{\psi T i}^{2}+\left(\alpha_{r i}^{c}-\alpha_{r i}\right)^{2}\right] \\
& \leq-2 K V_{\xi}+Q,
\end{aligned}
$$

where

$$
\begin{gathered}
K=\min \left\{\left[k_{c i}-\left(\sum_{j=1}^{N} 1.5 a_{i j}\right)\right],\right. \\
{\left[k_{U i}-\left(\sum_{j=1}^{N} 0.5 a_{i j}\right)\right],} \\
\left.\left[k_{\psi T i}-0.5-\sum_{j=1}^{N} 0.5 a_{i j} B_{1 i}^{2}\right]\right\}, \\
Q=\sum_{j=1}^{N} 0.5 a_{i j}\left(B_{1 i}^{2}+B_{3 i}^{2}\right)+\sum_{j=1}^{N} 0.5 B_{6 i}^{2}
\end{gathered}
$$

and $k_{c i}, k_{U i}$, and $k_{\psi T i}$ satisfy

$$
\begin{gathered}
k_{c i}>\left(\sum_{j=1}^{N} 1.5 a_{i j}\right), \\
k_{U i}>\left(\sum_{j=1}^{N} 0.5 a_{i j}\right), \\
k_{\psi T i}>0.5+\sum_{j=1}^{N} 0.5 a_{i j} B_{1 i}^{2} .
\end{gathered}
$$

With equality (93), one obtains

$$
V_{\xi}(t) \leq\left(V_{\xi}(0)-\frac{Q}{2 K}\right) e^{-2 K t}+\frac{Q}{2 K}:=\kappa .
$$

It could easily be found from equality (96) that $\left\|\boldsymbol{\xi}_{s i}\right\| \leq \sqrt{2 \kappa}$ for all $t \geq 0$.

Step 4 (implementation of the control objective). By noting that, with the boundedness of $\mathbf{E}_{S i}, \boldsymbol{\xi}_{S i}$, the signal $\mathbf{s}_{i}$ in (30) is bounded by $\chi+\sqrt{2 \kappa}$, define the vector as

$$
\begin{aligned}
\mathbf{S}(t) & =\left[\mathbf{s}_{1}^{T}(t), \ldots, \mathbf{s}_{M}^{T}(t)\right]^{T} \\
& =\left(\mathbf{L}_{1} \otimes \mathbf{I}_{2}\right) \mathbf{P}(t)+\left(\mathbf{L}_{2} \otimes \mathbf{I}_{2}\right) \mathbf{X}_{L}(t) .
\end{aligned}
$$

Then, the upper bound for the norm of $\mathbf{S}(t)$ can be obtained as below:

$$
\|\mathbf{S}(t)\| \leq \sum_{i=1}^{M}\left\|\mathbf{s}_{i}(t)\right\| \leq M(\chi+\sqrt{2 \kappa}) .
$$


Because $\mathbf{L}_{1}$ is invertible, equality (98) means that

$$
\begin{aligned}
\left\|\left(\mathbf{L}_{1}^{-1} \otimes \mathbf{I}_{2}\right) \mathbf{S}(t)\right\| & =\left\|\mathbf{P}(\mathbf{t})-\left(\mathbf{L}_{1}^{-1} \mathbf{L}_{2} \otimes \mathbf{I}_{2}\right) \mathbf{X}_{\mathrm{L}}(t)\right\| \\
& \leq \lambda_{\min }\left(\mathbf{L}_{1}^{-1}\right) M(\chi+\sqrt{2 \kappa}) \\
& :=S_{\max } .
\end{aligned}
$$

Thus, $\left\|\mathbf{p}_{i}-\mathbf{p}_{\text {di }}\right\|$ is bounded by $S_{\max }$ for all $t \geq 0$, where $\mathbf{p}_{d i}$ is defined in (25). Subsequently, the containment control objective (24) is solved.

Theorem 20. Consider a group of ships given by (6) and (7), and suppose Assumptions 3-5 hold. For each follower, consider the robust cooperative controllers (70) and (78) and FOCF (11); then, the tracking errors $\left\|\mathbf{p}_{i}-\mathbf{p}_{\text {di }}\right\|$ are globally uniformly ultimately bounded (GUUB), if the control parameters satisfy conditions (31), (91), and (95).

Remark 21. In $[16,17,44]$, the yaw velocity is required to be nonzero, which means that a straight-line reference cannot be tracked for ships. However, the PE condition is very restrictive from a practical point of view. By using a polar coordinate transformation, the proposed control strategy for ships can track not only straight-line paths but curved-line paths as well.

Remark 22. It is important to note that the traditional DSC method estimates the value of the virtual control law and its derivative only through first-order filter and fails to adjust its estimate through feedback. By contrast, FOCF method introduces compensation tracking error signal on the basis of traditional DSC method, realizes the real-time correction of virtual control law, and greatly improves the accuracy of the estimation.

Remark 23. The authors in [45] also adopt CFBP method to design the trajectory coordinated control strategy and use SOCF to estimate the virtual control law in the BP design procedure online. However, SOCF belongs to a class of secondorder systems, and it is more tedious to implement than FOCF. It should be mentioned that there are many deduction errors in [45]. In addition, the authors in [45] adopt the adaptive method to estimate the hydrodynamic parameters online in the dynamic design, but it is difficult to implement in practice. Therefore, in the paper, we refer to the design structure framework of literature [45], use first-order filter to estimate the virtual control laws, solve the containment control objective, and analyze the stability of the closed-loop system by using the input-output boundedness property of FOCF. Moreover, in the dynamic design, upper-to-up sliding mode method is used to design the actual control inputs of ship. Rather than online estimation of hydrodynamic parameters, the method only needs to use the boundedness of hydrodynamic parameters and external disturbances and has the advantages of simple structure and strong robustness.

3.4. Stability Analysis of Sway Motion. Since there is no available control input in the sway direction of the individual

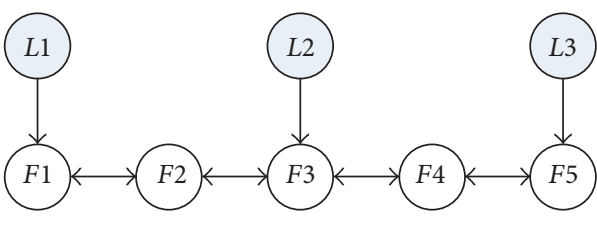

Figure 3: Communication topology.

TABLE 1: Parameters of ships.

\begin{tabular}{lcc}
\hline Parameter & Value & Unit \\
\hline$\widehat{m}_{11}$ & 25.8 & $\mathrm{~kg}$ \\
$\widehat{m}_{22}$ & 33.8 & $\mathrm{~kg}$ \\
$\widehat{m}_{33}$ & 2.76 & $\mathrm{~kg} \cdot \mathrm{m}^{2}$ \\
$\widehat{d}_{u}$ & 12 & $\mathrm{~kg} / \mathrm{s}$ \\
$\widehat{d}_{u 2}$ & 2.5 & $\mathrm{~kg} / \mathrm{m}$ \\
$\widehat{d}_{v}$ & 17 & $\mathrm{~kg} / \mathrm{s}$ \\
$\widehat{d}_{v 2}$ & 4.5 & $\mathrm{~kg} / \mathrm{m}$ \\
$\widehat{d}_{v r}$ & 0.2 & $\mathrm{~kg}$ \\
$\widehat{d}_{r}$ & 0.5 & $\mathrm{~kg} \cdot \mathrm{m}^{2} / \mathrm{s}$ \\
$\widehat{d}_{r 2}$ & 0.1 & $\mathrm{~kg} \cdot \mathrm{m}^{2}$ \\
$\widehat{d}_{r v}$ & 0.5 & $\mathrm{~kg}$ \\
\hline
\end{tabular}

ship (3), it is necessary to analyze the boundedness of the sway velocity $v_{i}$. Consider the following LFC:

$$
V_{5}=\frac{1}{2} m_{22 i} v_{i}^{2}
$$

From (100), the time derivative of $V_{5}$ can be obtained as

$$
\begin{aligned}
\dot{V}_{5} & =m_{22 i} v_{i} \dot{v}_{i} \\
& \leq-\left(d_{v i}+d_{v 2 i}\right) v_{i}^{2}-\left(u_{i} r_{i}+d_{v r i} r_{i}\right) v_{i}+\bar{\tau}_{w v i}\left|v_{i}\right| .
\end{aligned}
$$

Because $m_{11 i}, d_{v r i}, u_{i}$, and $v_{i}$ are bounded as shown in Section 3.2, one can obtain

$$
\dot{V}_{5} \leq-d_{v i} v_{i}^{2}+\varphi\left|v_{i}\right|
$$

where $\varphi=\max \left(m_{11 i}\left|u_{i} r_{i}\right|+d_{v r i}\left|r_{i}\right|\right)+\bar{\tau}_{w v i}$. It could be easily found that if $d_{v i}\left|v_{i}\right|>\varphi, \dot{V}_{5}<0$. That is to say, the sway velocity $v_{i}$ is uniformly ultimately bounded and will eventually converge to the invariant set $S_{v i}=\left\{v_{i} \in R\left|d_{v}\right| v_{i} \mid \leq \varphi\right\}$.

\section{Simulation Results}

To verify the effectiveness of the proposed robust cooperative scheme, a networked group that consists of the following five ships with three virtual leaders is considered. The communication topology among the ships is shown in Figure 3 with $F=\{1,2, \ldots, 5\}$ and $L=\{6,7,8\}$. It can be noted from Figure 3 that the network topology has a directed spanning tree. Assume that all the following ships have the identical structure and that mathematical model parameters are presented in Table 1. To satisfy conditions (31), (91), and (96), 
the cooperative control parameters for each following ship are chosen as follows:

$$
\begin{aligned}
& M_{11}=\widehat{m}_{11} \times 0.02, \\
& M_{22}=\widehat{m}_{22} \times 0.02 \text {, } \\
& M_{33}=\widehat{m}_{33} \times 0.02 \text {, } \\
& D_{v}=\widehat{d}_{v} \times 0.02, \\
& D_{v 2}=\widehat{d}_{v 2} \times 0.02 \text {, } \\
& D_{r}=\widehat{d}_{r} \times 0.02, \\
& D_{v}=\widehat{d}_{v} \times 0.02, \\
& D_{v 2}=\widehat{d}_{v 2} \times 0.02 \text {, } \\
& D_{r}=\widehat{d}_{r} \times 0.02 \text {, } \\
& D_{r 2}=\widehat{d}_{r 2} \times 0.02 \text {, } \\
& D_{v r}=\widehat{d}_{v r} \times 0.02 \text {, } \\
& H_{0}=\widehat{m}_{11} \times \frac{1.02}{\left(\widehat{m}_{22} \times 0.98\right)}, \\
& H_{u r}=\left(\frac{\widehat{m}_{11}^{2}}{\widehat{m}_{22}}\right) \times 0.02 \text {, } \\
& H_{v}=\left(\frac{\widehat{m}_{11} \widehat{d}_{v}}{\widehat{m}_{22}}\right) \times 0.02 \text {, } \\
& H_{v 2}=\left(\frac{\widehat{m}_{11} \widehat{d}_{v 2}}{\widehat{m}_{22}}\right) \times 0.02 \text {, } \\
& H_{r}=\frac{\widehat{m}_{11} \widehat{d}_{v r}}{\widehat{m}_{22}} \times 0.02 \text {, } \\
& k_{c i}=6 \text {, } \\
& k_{U i}=3 \text {, } \\
& k_{\psi T i}=5 \text {, } \\
& q_{1 i}=0.1, \\
& q_{2 i}=0.1 \text {, } \\
& \bar{\tau}_{w u i}=0.6, \\
& \bar{\tau}_{w v i}=0.6, \\
& \bar{\tau}_{w r i}=0.6 \text {, }
\end{aligned}
$$

and the FOCF parameter is selected as $\omega_{n}=10$. Next, four experiments will be presented to verify the effectiveness of the proposed algorithms.

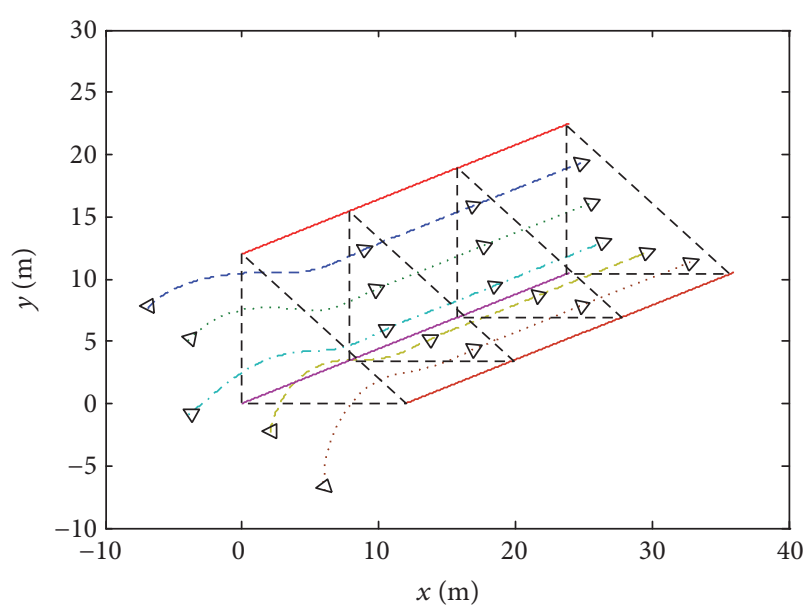

Figure 4: Motion trace of the group.

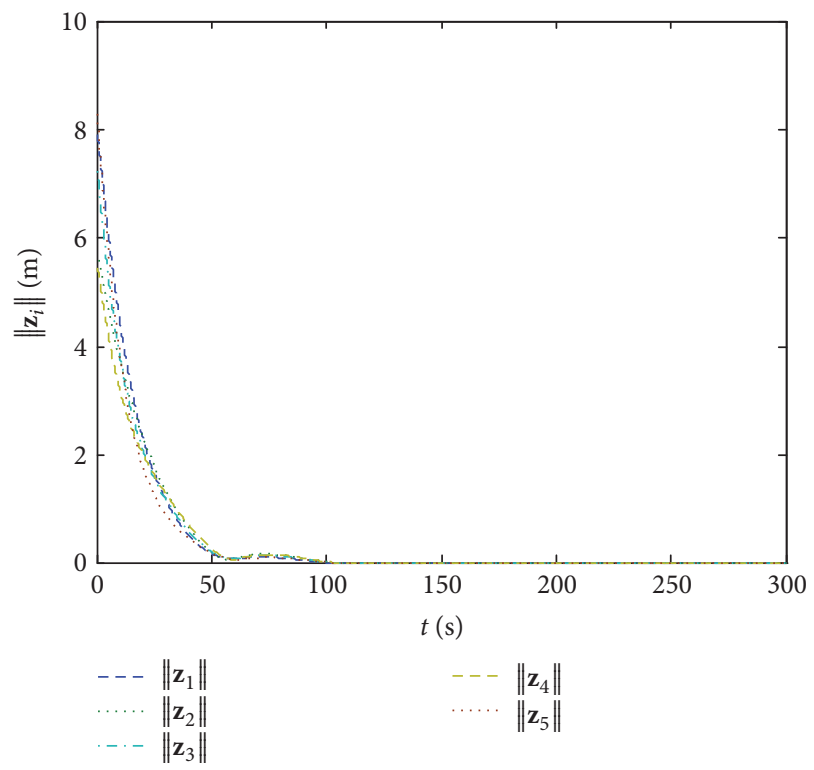

FIGURE 5: Error norms of the tracking errors $\mathbf{z}_{i}(i=1, \ldots, 5)$.

4.1. Straight-Line Tracking without EDPU. In this section, an example validates the proposed containment control strategy without considering the disturbances for the followers. The predefined trajectories of the virtual leaders are chosen as $\phi_{1}=[0.08 t ; 0.035 t+12], \phi_{2}=[0.08 t ; 0.035 t]$, and $\phi_{3}=$ $[0.08 t+12 ; 0.035 t]$. The initial follower states $\mathbf{X}_{i}=\left(x_{i} ; y_{i}\right.$; $\left.\psi_{i} ; u_{i} ; v_{i} ; r_{i}\right)$ are $\mathbf{X}_{1}=(-7 ;-7.5 ; \pi / 3 ; 0.05 ; 0.02 ; 0) ; \mathbf{X}_{2}=(-4 ;$ $5 ; \pi / 4 ; 0.02 ; 0.012 ; 0) ; \mathbf{X}_{3}=(-4 ;-1 ; \pi / 3 ; 0.04 ; 0.03 ; 0) ; \mathbf{X}_{4}=$ $(2 ;-2.5 ; \pi / 3 ; 0.01 ; 0.01 ; 0) ; \mathbf{X}_{5}=(6 ;-7 ; \pi / 2 ; 0.024 ; 0.015 ; 0)$. It is noted that when the trajectories of virtual leaders are straight lines, the desired trajectories are straight lines. The simulation results are shown in Figures 4-7. Also, the total simulation time is $200 \mathrm{~s}$. Figure 3 shows the motion traces of the followers. As shown in Figure 4, the five followers converge to the convex hull formed by those of the moving leaders. Figure 5 demonstrates that the norms of the tracking error for each follower converge asymptotically to zero as 


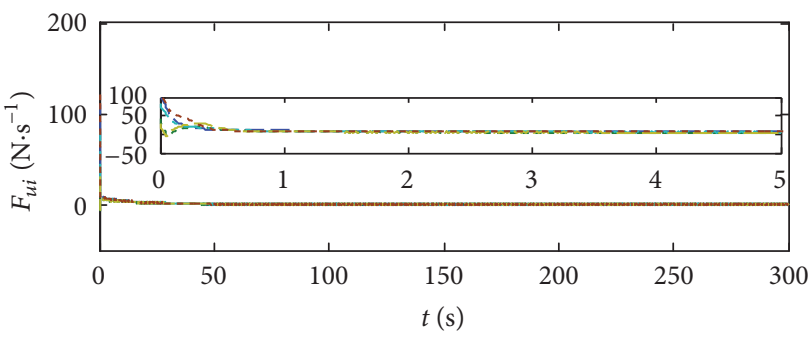

$---F_{u 1}$

-..- $F_{u 2}$

$-\cdots F_{u 3}$

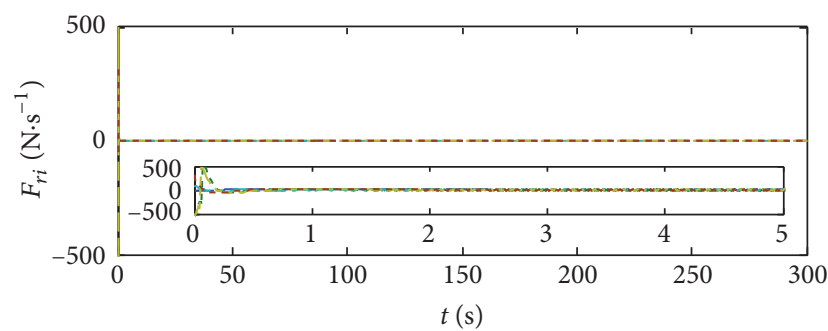

$--F_{r 1}$

$\cdots F_{r 2}$

$--F_{r 3}$

FIGURE 6: Control inputs for each follower.

$t \rightarrow \infty$, which reveals that the containment control objective (24) is solved. Figure 6 shows the actual control inputs of each follower. Figure 7 shows the time evolution of the FOCF estimated errors:

$$
\begin{gathered}
\tilde{e}_{U i}=\left|\boldsymbol{\alpha}_{U i}-\alpha_{U i}^{c}\right|, \\
\tilde{e}_{\psi T i}=\left|\boldsymbol{\alpha}_{\psi T i}-\alpha_{\psi T i}^{c}\right|, \\
\tilde{e}_{r i}=\left|\alpha_{r i}-\alpha_{r i}^{c}\right| .
\end{gathered}
$$

It is obvious from Figure 7 that FOCF can accurately estimate virtual control laws.

4.2. Straight-Line Tracking with EDPU. In this section, the robust containment control strategy in the presence of the disturbances is also examined. The trajectories of the virtual leaders are chosen as Section 4.1. The EDPU for the followers in the inertial reference frame $\{I\}$ are assumed to be

$$
\begin{aligned}
& \tau_{w u i}(t)=0.28 \sin \left(0.016 t+\frac{\pi}{3}\right)+0.02(\mathrm{~N}), \\
& \tau_{w v i}(t)=0.014 \cos \left(0.01 t+\frac{\pi}{4}\right)+0.04(\mathrm{~N}), \\
& \tau_{w r i}(t)=0.042 \sin \left(0.008 t+\frac{\pi}{4}\right)+0.008(\mathrm{~N} \cdot \mathrm{m}),
\end{aligned}
$$

where $i=1,2, \ldots, 5$. The initial follower states are chosen as Section 4.1. The model parameters, used for simulation
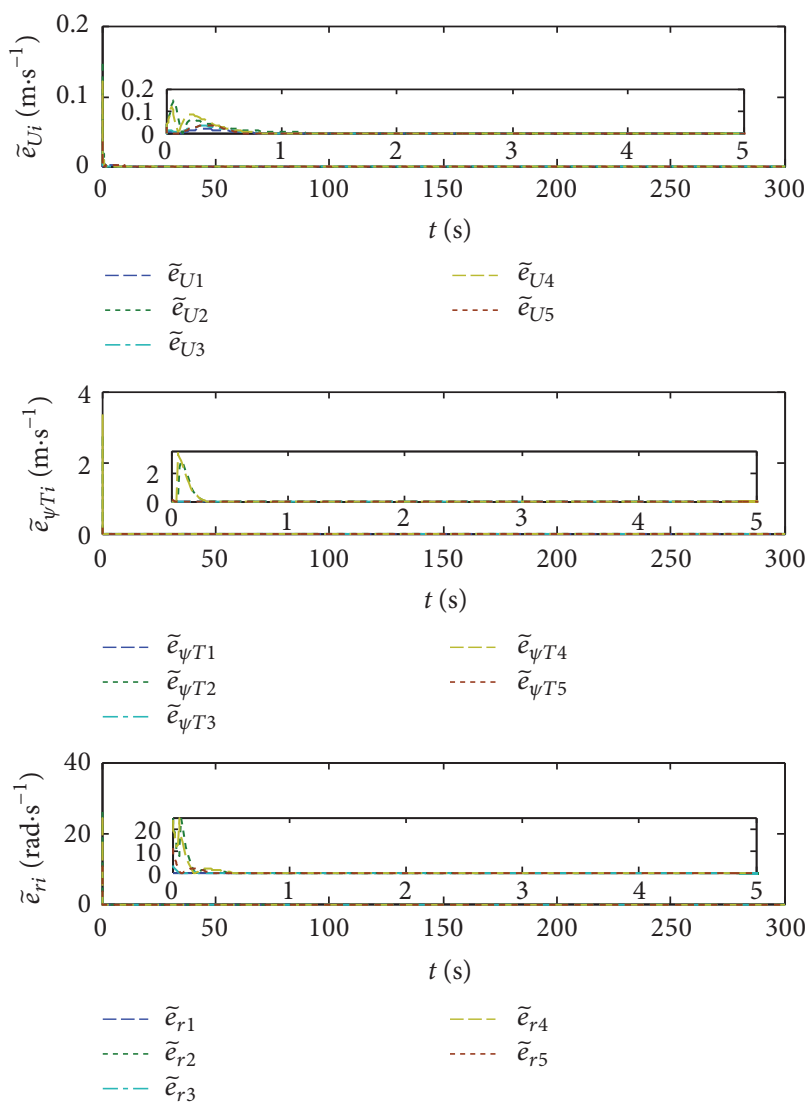

Figure 7: Tracking errors $\widetilde{e}_{U i}, \widetilde{e}_{\psi T i}, \widetilde{e}_{r i}(i=1, \ldots, 5)$ for FOCF.

experiment, are given in the following, assuming that the model parameters are $1.5 \%$ parameter perturbation:

$$
\begin{aligned}
m_{11} & =\widehat{m}_{11}[1+0.015 \sin (0.1 \mathrm{t})](\mathrm{kg}) \\
m_{22} & =\widehat{m}_{22}\left[1+0.015 \cos \left(0.1 t+\frac{\pi}{3}\right)\right](\mathrm{kg}) \\
m_{33} & =\widehat{m}_{33}\left[1+0.015 \sin \left(0.1 t+\frac{\pi}{6}\right)\right]\left(\mathrm{kg} \cdot \mathrm{m}^{2}\right) \\
d_{u} & =\widehat{d}_{u}[1-0.015 \sin (0.1 t)](\mathrm{kg} / \mathrm{s}) \\
d_{u 2} & =\widehat{d}_{u 2}[1+0.015 \sin (0.1 \mathrm{t})](\mathrm{kg} / \mathrm{m}) \\
d_{v} & =\widehat{d}_{v}[1+0.015 \sin (0.1 t)](\mathrm{kg} / \mathrm{s}), \\
d_{v 2} & =\widehat{d}_{v 2}\left[1+0.015 \cos \left(0.1 t-\frac{\pi}{4}\right)\right]\left(\mathrm{kg} / \mathrm{m}^{2}\right) \\
d_{v r} & =\widehat{d}_{v r}\left[1+0.015 \cos \left(0.1 t+\frac{\pi}{3}\right)\right](\mathrm{kg}), \\
d_{r} & =\widehat{d}_{r}\left[1+0.015 \sin \left(0.1 t+\frac{\pi}{6}\right)\right]\left(\mathrm{kg} \cdot \mathrm{m}^{2}\right) \\
d_{r 2} & =\widehat{d}_{r 2}[1-0.015 \sin (0.1 t)]\left(\mathrm{kg} \cdot \mathrm{m}^{2} / \mathrm{s}\right), \\
d_{r v} & =\widehat{d}_{r v}[1+0.015 \cos (0.1 t)](\mathrm{kg}) .
\end{aligned}
$$




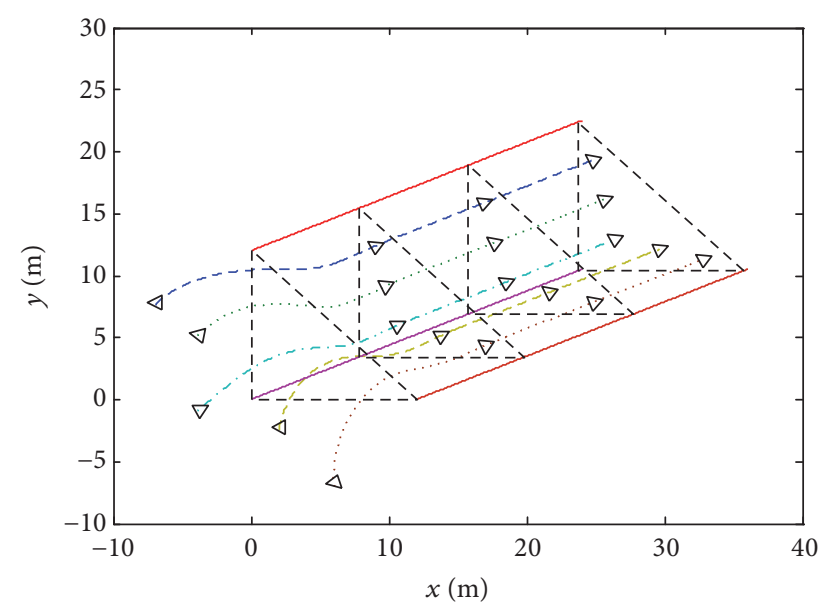

FIGURE 8: Motion trace of the group.

The simulation results are depicted in Figures 8-11, which exhibit almost the same control performance as those given in Section 4.1. Figure 8 shows the motion traces of the five followers, from which it is evident that the followers also converge to the convex hull formed by those of the moving leaders. The norms of the tracking errors are plotted in Figure 9, and it reveals that the tracking error for each follower are bounded to a small neighborhood of the origin as $t \rightarrow \infty$. It is clearly seen from Figures 5 and 9 that the proposed controller yields slightly worse performance than the previous section due to the EDPU. However, the tracking errors are bounded, which means that the tracking errors for each follower are GUUB as proved in Theorem 20. Figure 10 shows the actual control inputs applied to each follower under the EDPU, whereas Figure 11 shows the time evolution of the FOCF estimated errors. Therefore, from the simulation results in this section, it is obvious to conclude that the proposed containment control strategy is also practical when each follower is exposed to the EDPU. Also, it is clear that the proposed containment control strategy for each follower can track straight lines without assuming the PE condition.

4.3. Curve Tracking without EDPU. In this section, the predefined trajectories of the virtual leaders are curved trajectories. This experiment validates the control strategy without considering the EDPU. The initial follower states $\mathbf{X}_{i}=$ $\left(x_{i} ; y_{i} ; \psi_{i} ; u_{i} ; v_{i} ; r_{i}\right)$ are $\mathbf{X}_{1}=(-14.5 ;-5 ; \pi / 3 ; 0.01 ; 0.02 ; 0)$; $\mathbf{X}_{2}=(-12 ;-7 ; \pi / 4 ; 0.02 ; 0.012 ; 0) ; \mathbf{X}_{3}=(-8 ;-6 ; \pi / 6 ; 0.02 ;$ $0.03 ; 0) ; \mathbf{X}_{4}=(-6 ;-7 ; \pi / 3 ; 0.01 ; 0.01 ; 0) ; \mathbf{X}_{5}=(-4 ;-5 ; \pi / 2.5 ;$ $0 ; 0.015 ; 0)$. In the following simulation, the curved trajectories of virtual leaders are chosen as

$$
\begin{aligned}
\phi_{2} & =\left[-8 \cos \left(\frac{\pi t}{200}\right) ; 8 \sin \left(\frac{\pi t}{200}\right)\right], \\
\phi_{i} & =\phi_{2}+\mathbf{R}\left(\psi_{d}\right) \mathbf{l}_{i},
\end{aligned}
$$

where $i=1,3 ; \mathbf{R}(\cdot)=[\cos (\cdot),-\sin (\cdot) ; \sin (\cdot), \cos (\cdot)] ; \psi_{d}=$ $\operatorname{atan} 2[\pi / 25 \cos (\pi t / 200), \pi / 25 \sin (\pi t / 200)] ; \mathbf{l}_{1}=[-4 ; 4], \mathbf{l}_{2}=$ $[-4 ;-4]$. The simulation results are plotted in Figures $12-15$. Figure 12 illustrates the motion traces of the five followers. It

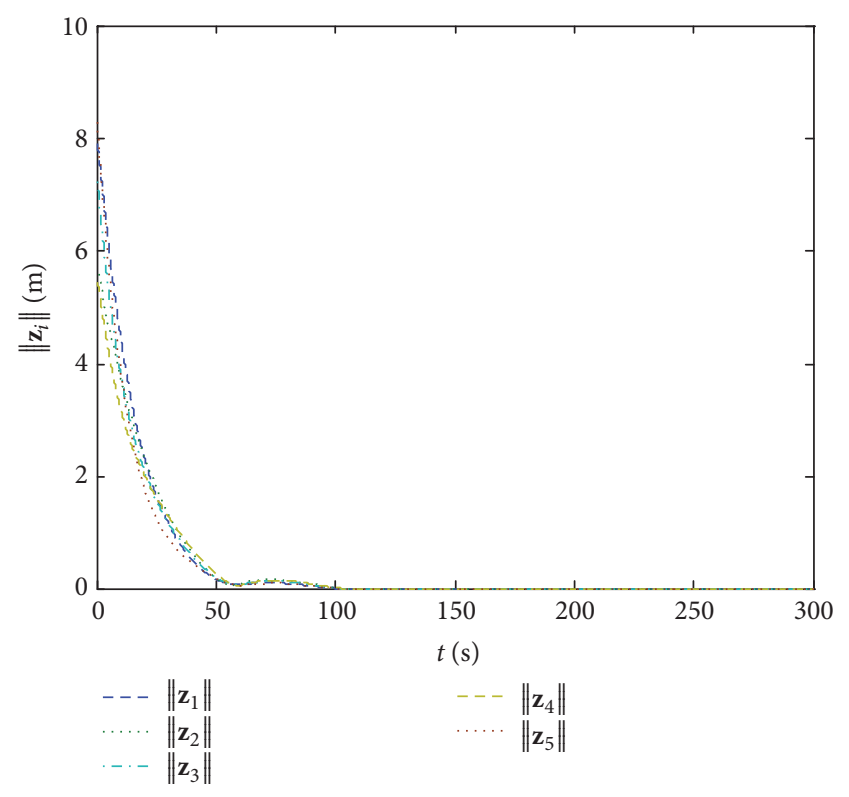

FIGURE 9: Error norms of the tracking errors $\mathbf{z}_{i}(i=1, \ldots, 5)$.
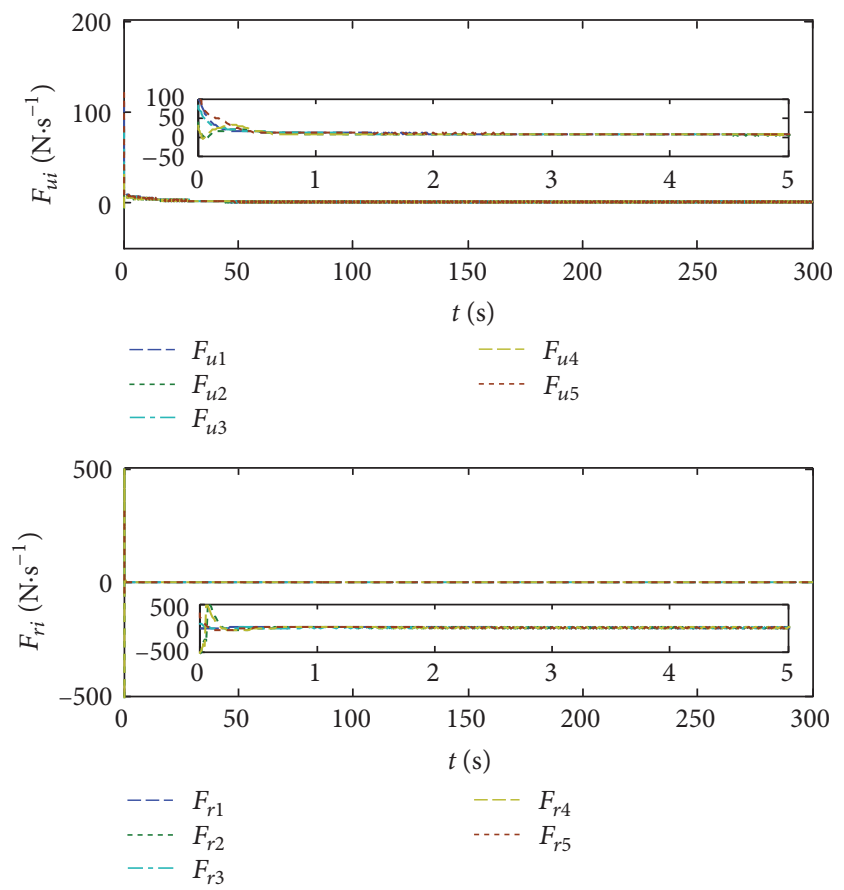

FIGURE 10: Control inputs for each follower.

is obviously seen that the control strategy can also track the curved trajectories defined by the virtual leaders. The norms of the tracking errors are plotted in Figure 13. From this figure, we can observe that the tracking errors converge to a small neighborhood of the origin with fast convergence rate. The responses of the control inputs are displayed in Figure 14. In Figure 15, FOCF estimated errors converge to nearly zero.

4.4. Curve Tracking with EDPU. In this section, the simulation experiments are presented, which concern the robustness properties of the containment control law to the EDPU. 

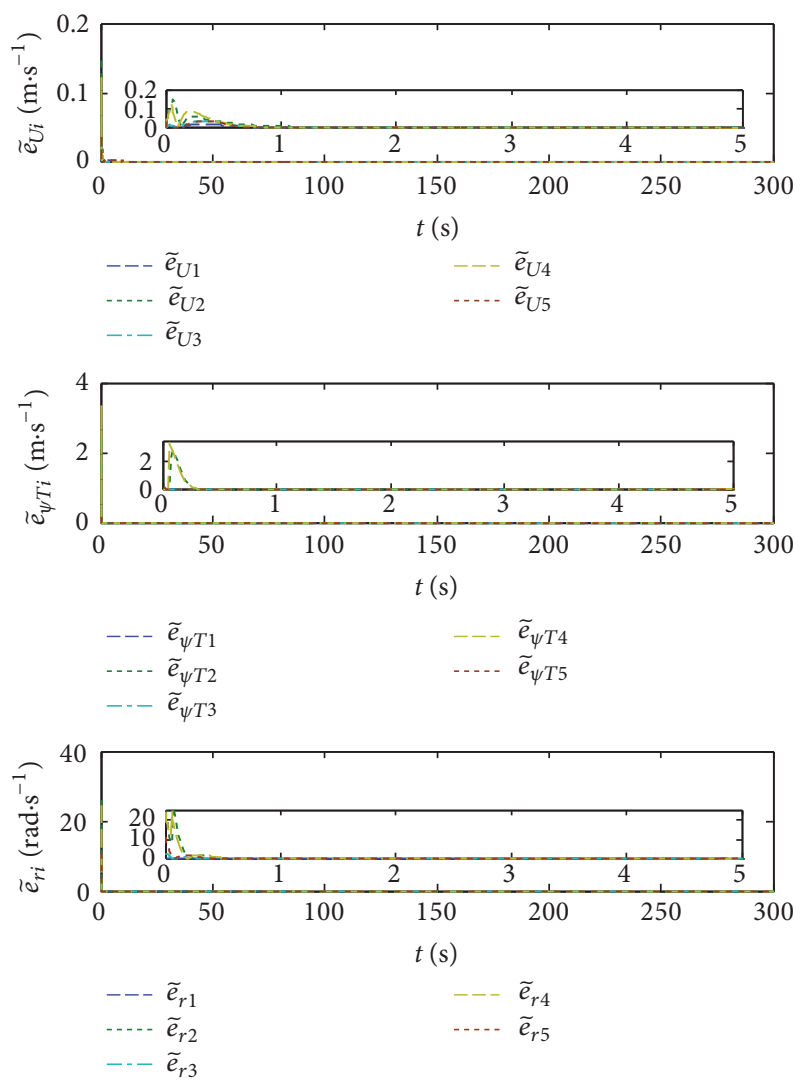

FIgURE 11: Tracking errors $e_{U i}, e_{\psi T i}$, and $e_{r i}(i=1, \ldots, 5)$ for FOCF.

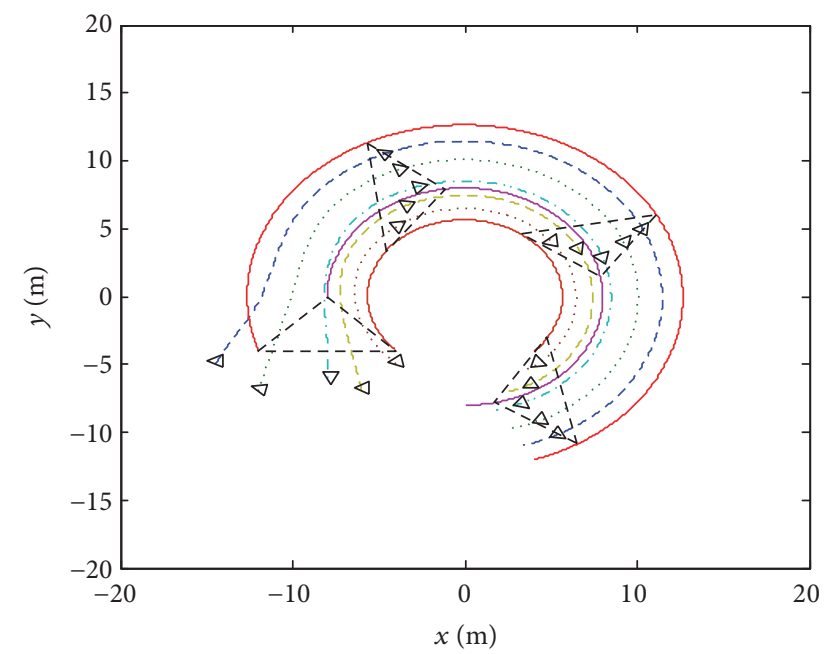

FIGURE 12: Motion trace of the group.

The initial states of the followers and the curved predefined trajectories of leaders are the same as the previous section. These simulation results are demonstrated in Figures 16-19. Figure 16 shows the actual trajectories of the followers. The norms of the tracking errors are plotted in Figure 17. As seen from Figures 16 and 17, the containment control laws can force the followers to converge into the triangular region in the presence of the EDPU, and the norms of the tracking

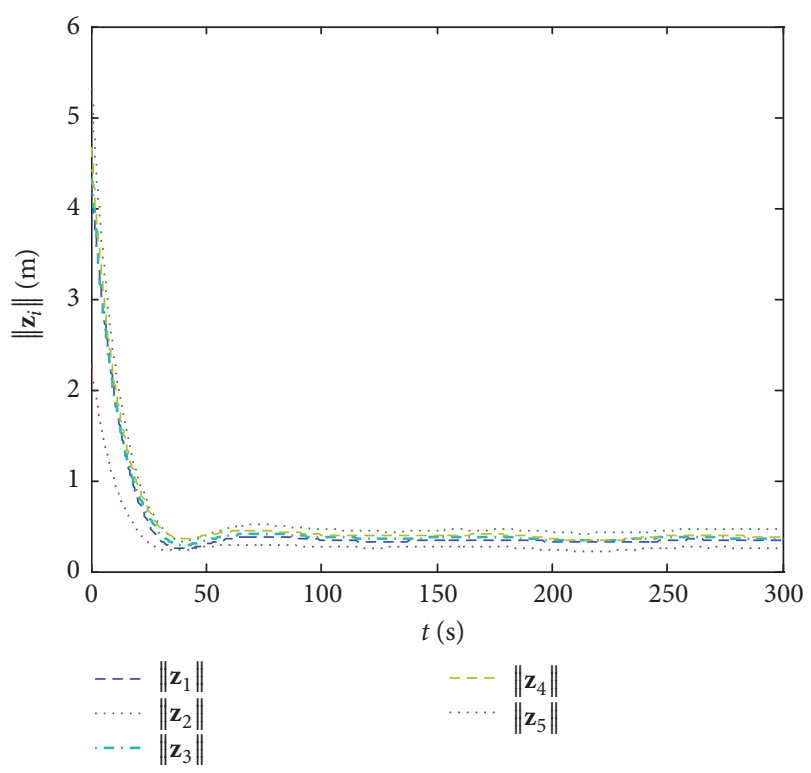

FIGURE 13: Error norms of the tracking errors $\mathbf{z}_{i}(i=1, \ldots, 5)$.

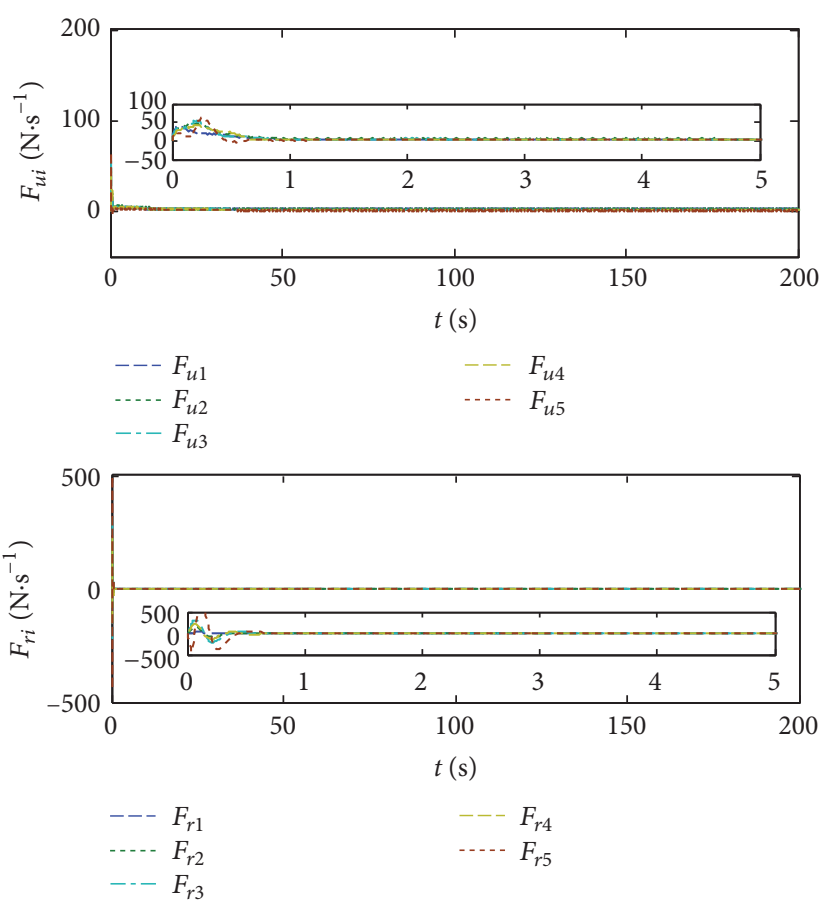

FIGURE 14: Control inputs for each follower.

errors are all less than $0.75 \mathrm{~m}$. In Figure 18, the control forces $F_{u i}$ and $F_{r i}$ are shown. Figure 19 illustrates the responses of the FOCF estimated errors.

In summary, from Figures 4-19, we can see that the proposed containment control law in this paper provided acceptable results as proven in Theorem 20 and all the tracking errors of the closed-loop system converge to a small neighborhood of the origin as time approaches infinity. 

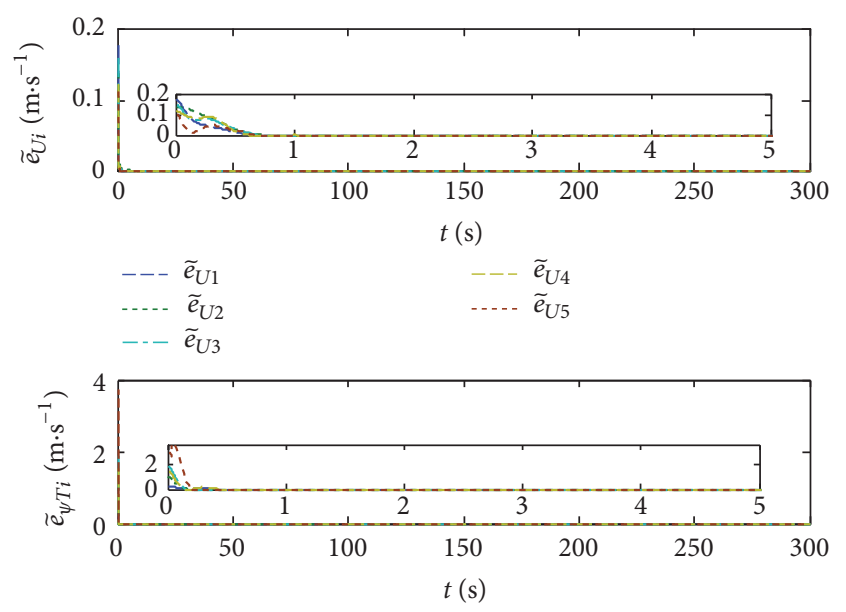

$$
\begin{array}{llll} 
& \tilde{e}_{\psi T 1} & & \\
\ldots-- & \tilde{e}_{\psi T 4} \\
\ldots & \tilde{e}_{\psi T 2} & \ldots & \tilde{e}_{\psi T 5} \\
-- & \tilde{e}_{\psi T 3} & &
\end{array}
$$

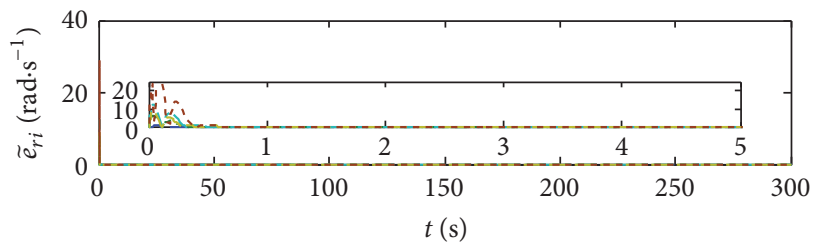

$$
\begin{array}{llll}
--- & \widetilde{e}_{r 1} & --- & \tilde{e}_{r 4} \\
-\cdots & \widetilde{e}_{r 2} & \ldots- & \tilde{e}_{r 5} \\
--- & \widetilde{e}_{r 3} & &
\end{array}
$$

FIGURE 15: Tracking errors $e_{U i}, e_{\psi T i}$, and $e_{r i}(i=1, \ldots, 5)$ for FOCF.

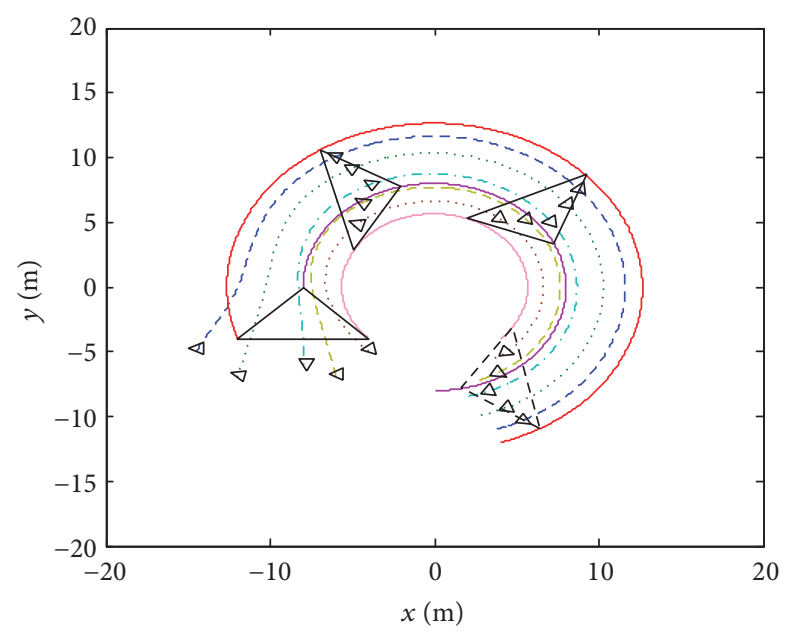

FIGURE 16: Motion trace of the group.

\section{Conclusions}

(1) Because the sway force is unavailable, the underactuated model is transformed to a strict-feedback form by introducing a polar coordinate transformation. In the kinematic loop design, a virtual controller is designed on the basis of the results from graph theory so as to achieve the control objective.

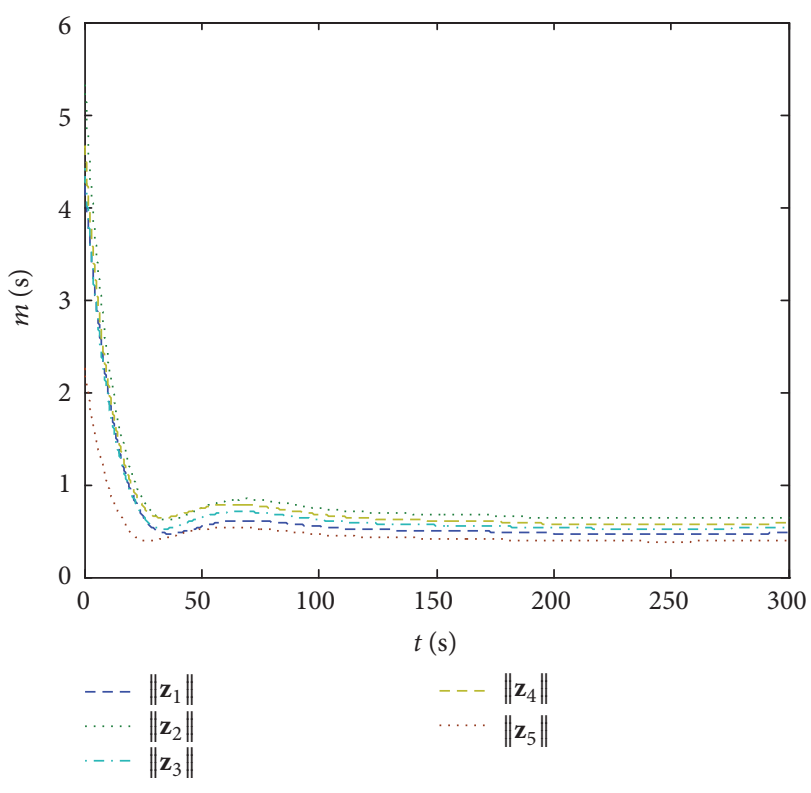

FIgURE 17: Error norms of the tracking errors $\mathbf{z}_{i}(i=1, \ldots, 5)$.
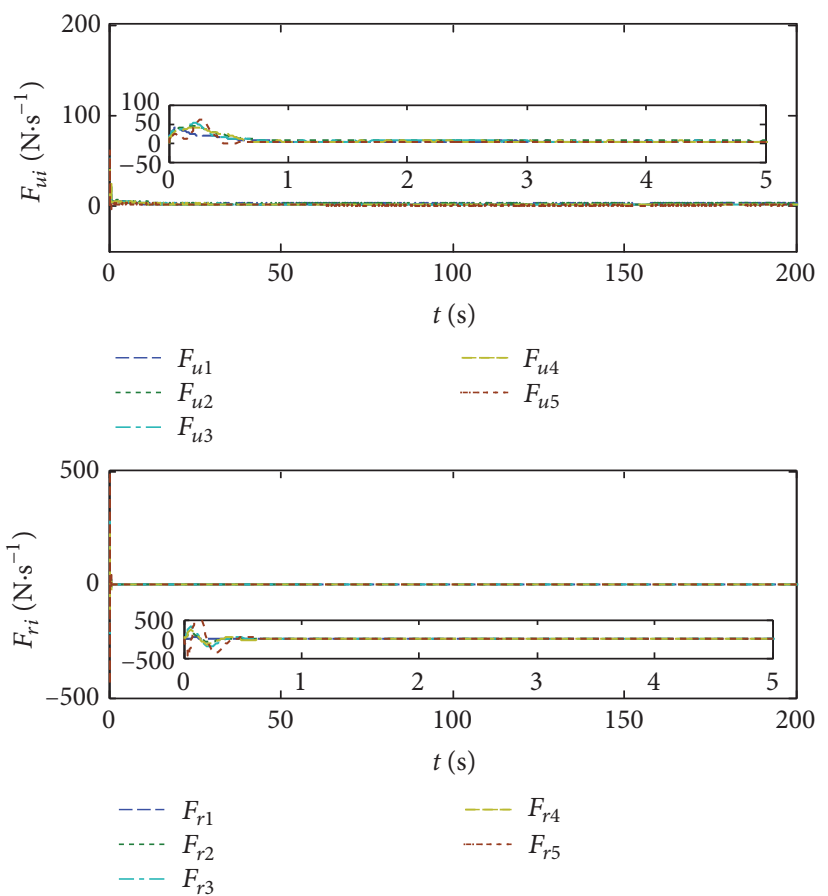

FIGURE 18: Control inputs for each follower.

(2) To avoid the problem of "explosion of complexity" from the standard BP method, FOCF is introduced to estimate the virtual control laws and their derivatives. In addition, a compensating signal is introduced to compensate the nonlinear terms in BP design.

(3) Under the consideration that each follower is exposed to the EDPU, the actual control inputs are presented by using the SMC method in the dynamic loop design, which can guarantee that all the signals of the closed-loop system are 


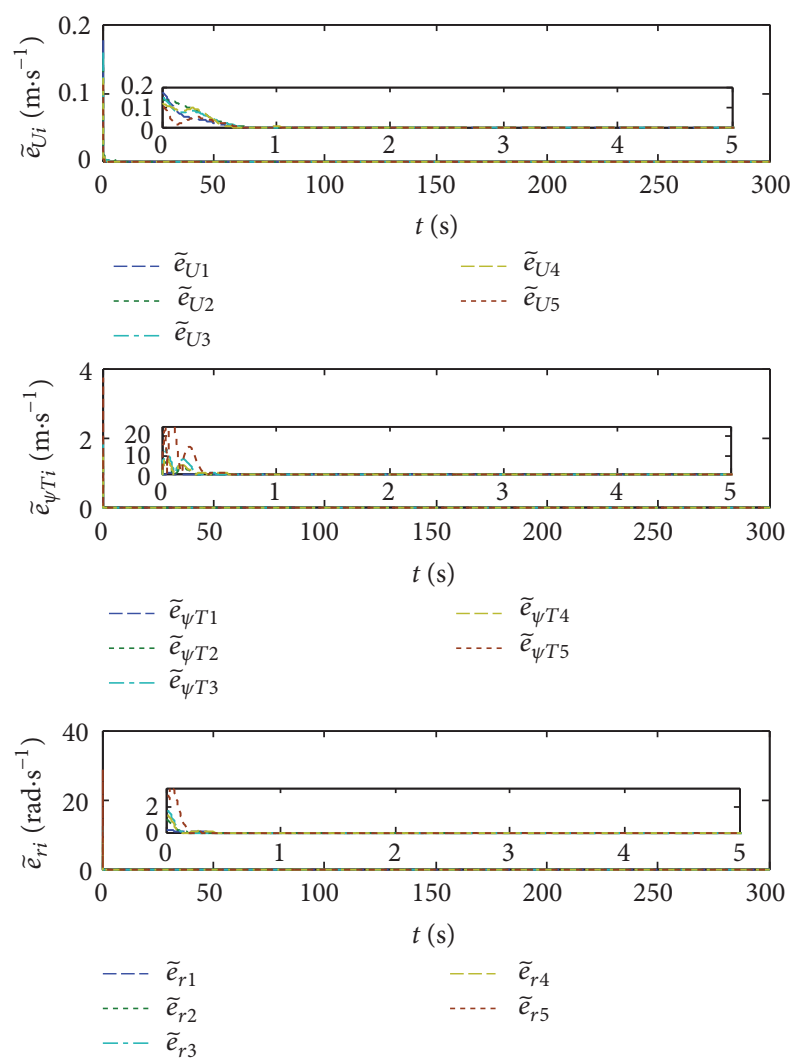

FIGURE 19: Tracking errors $e_{U i}, e_{\psi T i}$, and $e_{r i}(i=1, \ldots, 5)$ for FOCF.

GUUB and all the tracking errors eventually converge to a small neighborhood of the equilibrium point.

(4) Future works will be required to account for communication constraint among ships, such as packets dropouts and constant or time-varying communication delays as well as switching communication topology.

\section{Conflicts of Interest}

The authors declare that there are no conflicts of interest regarding the publication of this paper.

\section{Acknowledgments}

This work is supported partially by the National Natural Science Foundation of China (no. 51379044), the Fundamental Research Funds for the Central Universities (no. HEUCFX41304), and the Natural Science Foundation of Heilongjiang Province (no. F200916).

\section{References}

[1] H. A. Poonawala, A. C. Satici, H. Eckert, and M. W. Spong, "Collision-free formation control with decentralized connectivity preservation for nonholonomic-wheeled mobile robots," IEEE Transactions on Control of Network Systems, vol. 2, no. 2, pp. 122-130, 2015.
[2] Q. Yang, H. Fang, J. Chen, and X. Wang, "Distributed observerbased coordination for multiple Lagrangian systems using only position measurements," IET Control Theory \& Applications, vol. 8, no. 17, pp. 2102-2114, 2014.

[3] P. Lu, C. Gan, and X. Liu, "Finite-time distributed cooperative attitude control for multiple spacecraft with actuator saturation," IET Control Theory \& Applications, vol. 8, no. 18, pp. 21862198, 2014.

[4] Z. Peng, D. Wang, Z. Chen, X. Hu, and W. Lan, "Adaptive dynamic surface control for formations of autonomous surface vehicles with uncertain dynamics," IEEE Transactions on Control Systems Technology, vol. 21, no. 2, pp. 513-520, 2013.

[5] K. Shojaei, "Leader-follower formation control of underactuated autonomous marine surface vehicles with limited torque," Ocean Engineering, vol. 105, pp. 196-205, 2015.

[6] J. Almeida, C. Silvestre, and A. M. Pascoal, "Cooperative control of multiple surface vessels with discrete-time periodic communications," International Journal of Robust and Nonlinear Control, vol. 22, no. 4, pp. 398-419, 2012.

[7] M. Fu and J. Jiao, "A hybrid approach for coordinated formation control of multiple surface vessels," Mathematical Problems in Engineering, vol. 2013, Article ID 794284, 8 pages, 2013.

[8] F. Arrichiello, S. Chiaverini, and T. I. Fossen, "Formation control of underactuated surface vessels using the null-spaee-based behavioral control," in Proceedings of the IEEE/RSJ International Conference on Intelligent Robots and Systems (IROS '06), pp. 5942-5947, October 2006.

[9] I. F. Ihle, M. Arcak, and T. I. Fossen, "Passivity-based designs for synchronized path-following," Automatica, vol. 43, no. 9, pp. 1508-1518, 2007.

[10] M.-Y. Fu and J.-F. Jiao, "Passive coordinated formation control for vessels based on virtual leader," Acta Automatica Sinica, vol. 40, no. 3, pp. 439-448, 2014.

[11] Z. Peng, D. Wang, T. Li, and Z. Wu, "Leaderless and leaderfollower cooperative control of multiple marine surface vehicles with unknown dynamics," Nonlinear Dynamics, vol. 74, no. 1-2, pp. 95-106, 2013.

[12] E. Børhaug, A. Pavlov, E. Panteley, and K. Y. Pettersen, "Straight line path following for formations of underactuated marine surface vessels," IEEE Transactions on Control Systems Technology, vol. 19, no. 3, pp. 493-506, 2011.

[13] J. Ghommam and F. Mnif, "Coordinated path-following control for a group of underactuated surface vessels," IEEE Transactions on Industrial Electronics, vol. 56, no. 10, pp. 3951-3963, 2009.

[14] H. Wang, D. Wang, Z. Peng, and W. Wang, "Adaptive dynamic surface control for cooperative path following of underactuated marine surface vehicles via fast learning," IET Control Theory o Applications, vol. 7, no. 15, pp. 1888-1898, 2013.

[15] E. Kyrkjebø, K. Y. Pettersen, M. Wondergem, and H. Nijmeijer, "Output synchronization control of ship replenishment operations: theory and experiments," Control Engineering Practice, vol. 15, no. 6, pp. 741-755, 2007.

[16] W. Dong and J. A. Farrell, "Formation control of multiple underactuated surface vessels," IET Control Theory \& Applications, vol. 2, no. 12, pp. 1077-1085, 2008.

[17] W. Dong, "Cooperative control of underactuated surface vessels," IET Control Theory \& Applications, vol. 4, no. 9, pp. 15691580, 2010.

[18] F. Fahimi, "Non-linear model predictive formation control for groups of autonomous surface vessels," International Journal of Control, vol. 80, no. 8, pp. 1248-1259, 2007. 
[19] B. S. Park, "Adaptive formation control of underactuated autonomous underwater vehicles," Ocean Engineering, vol. 96, pp. 1-7, 2015.

[20] G. Zhang, X. Zhang, and Y. Zheng, "Adaptive neural pathfollowing control for underactuated ships in fields of marine practice," Ocean Engineering, vol. 104, pp. 558-567, 2015.

[21] J. Ghommam, F. Mnif, A. Benali, and N. Derbel, "Asymptotic backstepping stabilization of an underactuated surface vessel," IEEE Transactions on Control Systems Technology, vol. 14, no. 6, pp. 1150-1157, 2006.

[22] K. D. Do, "Practical formation control of multiple underactuated ships with limited sensing ranges," Robotics and Autonomous Systems, vol. 59, no. 6, pp. 457-471, 2011.

[23] S. J. Yoo, "Distributed adaptive containment control of uncertain nonlinear multi-agent systems in strict-feedback form," Automatica, vol. 49, no. 7, pp. 2145-2153, 2013.

[24] Z. Peng, D. Wang, W. Wang, and L. Liu, "Containment control of networked autonomous underwater vehicles: a predictorbased neural DSC design," ISA Transactions, vol. 59, pp. 160-171, 2015.

[25] Y. Cao and W. Ren, "Containment control with multiple stationary or dynamic leaders under a directed interaction graph," in Proceedings of the 48th IEEE Conference on Decision and Control, pp. 3014-3019, IEEE, Shanghai, China, December 2009.

[26] Y. Cao, D. Stuart, W. Ren, and Z. Meng, "Distributed containment control for multiple autonomous vehicles with doubleintegrator dynamics: algorithms and experiments," IEEE Transactions on Control Systems Technology, vol. 19, no. 4, pp. 929938, 2011.

[27] H. Su and M. Z. Chen, "Multi-agent containment control with input saturation on switching topologies," IET Control Theory \& Applications, vol. 9, no. 3, pp. 399-409, 2015.

[28] A. Abdessameud, I. G. Polushin, and A. Tayebi, "Containment control for networked Lagrangian systems under a directed graph and communication constraints," in Proceedings of the 53rd IEEE Annual Conference on Decision and Control (CDC '14), pp. 2938-2943, IEEE, December 2014.

[29] T. Kheowree and S. Kuntanapreeda, "Adaptive dynamic surface control of an electrohydraulic actuator with friction compensation," Asian Journal of Control, vol. 17, no. 3, pp. 855-867, 2015.

[30] J. A. Farrell, M. Polycarpou, M. Sharma, and W. Dong, "Command filtered backstepping," IEEE Transactions on Automatic Control, vol. 54, no. 6, pp. 1391-1395, 2009.

[31] W. Dong, J. A. Farrell, M. M. Polycarpou, V. Djapic, and M. Sharma, "Command filtered adaptive backstepping," IEEE Transactions on Control Systems Technology, vol. 20, no. 3, pp. 566-580, 2012.

[32] B. Xu, S. Wang, D. Gao, Y. Zhang, and Z. Shi, "Command filter based robust nonlinear control of hypersonic aircraft with magnitude constraints on states and actuators," Journal of Intelligent and Robotic Systems: Theory and Applications, vol. 73, no. 1-4, pp. 233-247, 2014.

[33] J. Yu, P. Shi, W. Dong, and H. Yu, "Observer and commandfilter-based adaptive fuzzy output feedback control of uncertain nonlinear systems," IEEE Transactions on Industrial Electronics, vol. 62, no. 9, pp. 5962-5970, 2015.

[34] B. Miao and T. Li, "A novel neural network-based adaptive control for a class of uncertain nonlinear systems in strictfeedback form," Nonlinear Dynamics, vol. 79, no. 2, pp. 10051013, 2015.
[35] W. Ren and Y. Cao, Distributed Coordination of Multi-Agent Networks: Emergent Problems, Models, and Issues, Springer, 2010.

[36] Y. Cao, W. Ren, and M. Egerstedt, "Distributed containment control with multiple stationary or dynamic leaders in fixed and switching directed networks," Automatica, vol. 48, no. 8, pp. 1586-1597, 2012.

[37] T. I. Fossen, Handbook of Marine Craft Hydrodynamics and Motion Control, John Wiley \& Sons, West Sussex, UK, 2011.

[38] X. Qi, "Adaptive coordinated tracking control of multiple autonomous underwater vehicles," Ocean Engineering, vol. 91, pp. 84-90, 2014.

[39] D. Wang, "Neural network-based adaptive dynamic surface control of uncertain nonlinear pure-feedback systems," International Journal of Robust and Nonlinear Control, vol. 21, no. 5, pp. 527-541, 2011.

[40] J. Li, W. Ren, and S. Xu, "Distributed containment control with multiple dynamic leaders for double-integrator dynamics using only position measurements," IEEE Transactions on Automatic Control, vol. 57, no. 6, pp. 1553-1559, 2012.

[41] X. Wang, Y. Hong, and H. Ji, "Adaptive multi-agent containment control with multiple parametric uncertain leaders," Automatica, vol. 50, no. 9, pp. 2366-2372, 2014.

[42] J. Mei, W. Ren, B. Li, and G. Ma, "Containment control for networked unknown Lagrangian systems with multiple dynamic leaders under a directed graph," in Proceedings of the 1st American Control Conference (ACC '13), pp. 522-527, Washington, DC, USA, June 2013.

[43] V. Djapic, J. Farrell, and W. Dong, "Land vehicle control using a command filtered backstepping approach," in Proceedings of the American Control Conference (ACC '08), pp. 2461-2466, Seattle, Wash, USA, June 2008.

[44] Y.-L. Liao, M.-J. Zhang, L. Wan, and Y. Li, “Trajectory tracking control for underactuated unmanned surface vehicles with dynamic uncertainties," Journal of Central South University, vol. 23, no. 2, pp. 370-378, 2016.

[45] J. Ghommam and M. Saad, "Backstepping-based cooperative and adaptive tracking control design for a group of underactuated AUVs in horizontal plan," International Journal of Control, vol. 87, no. 5, pp. 1076-1093, 2014. 


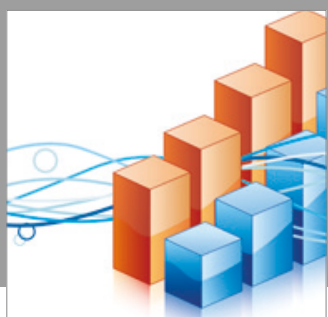

Advances in

Operations Research

vatersals

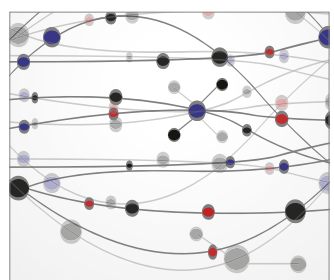

\section{The Scientific} World Journal
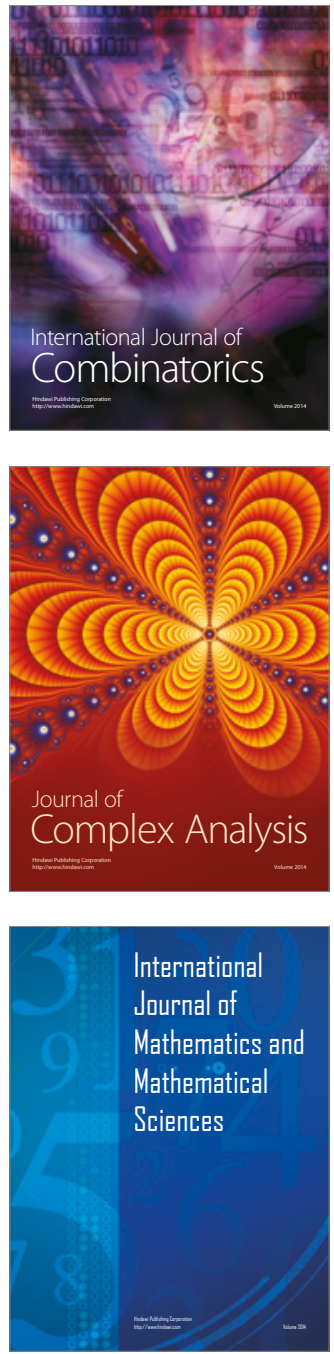
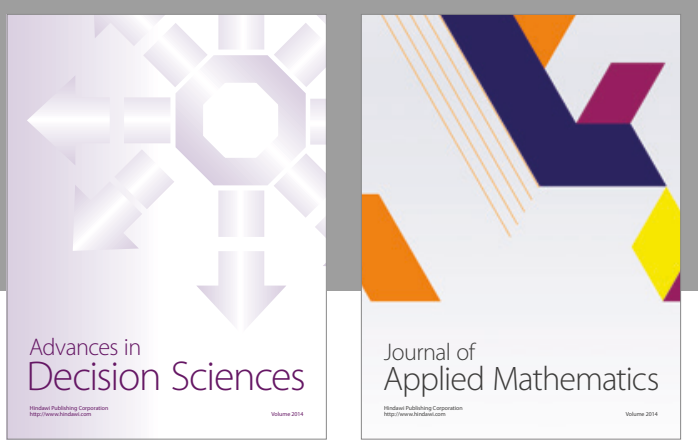

Algebra

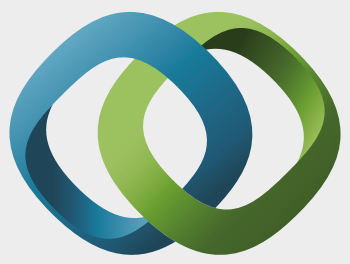

\section{Hindawi}

Submit your manuscripts at

https://www.hindawi.com
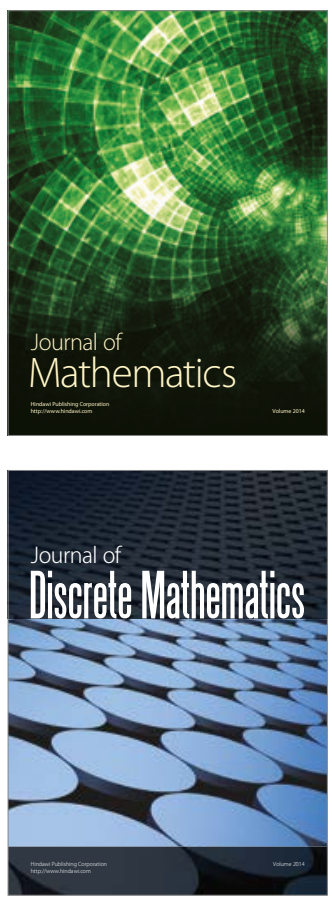

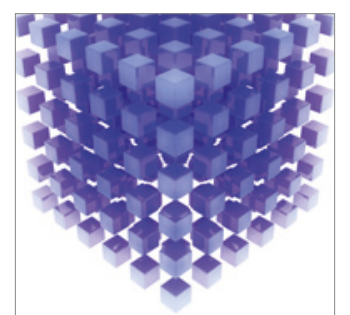

Mathematical Problems in Engineering
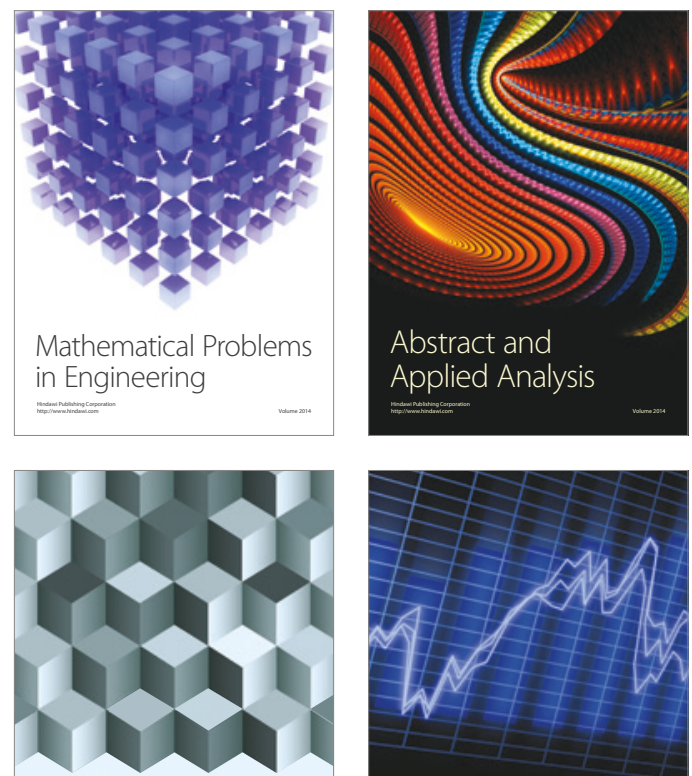

Journal of

Function Spaces

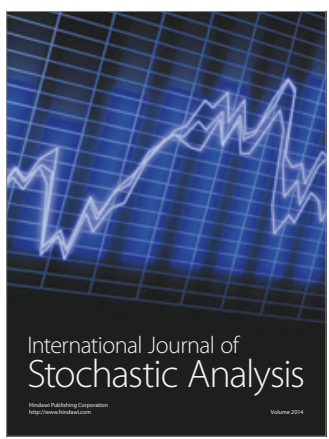

Probability and Statistics
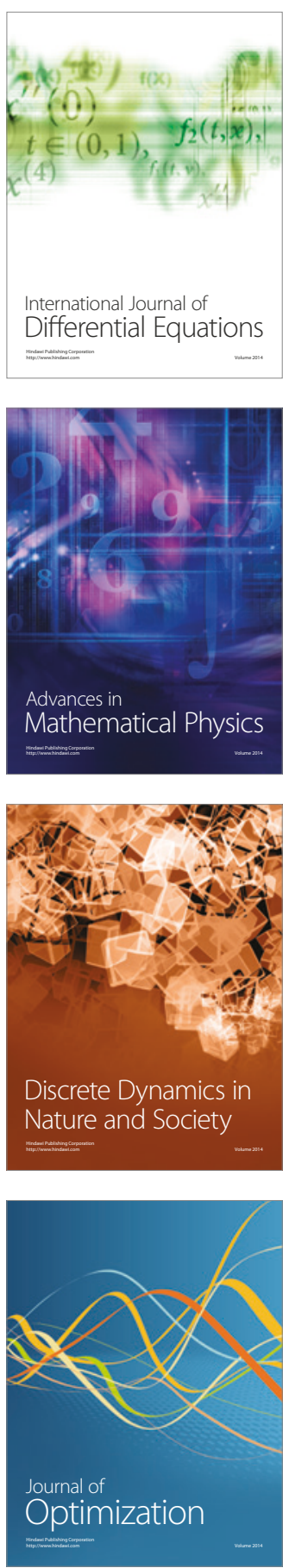